\title{
The untold stories of the speech gene, the FOXP2 cancer gene
}

\author{
Maria Jesus Herrero ${ }^{1, *}$ and Yorick Gitton ${ }^{2, *}$ \\ ${ }^{1}$ Center for Neuroscience Research, Children's National Medical Center, NW, Washington, DC, USA \\ 2 Sorbonne University, INSERM, CNRS, Vision Institute Research Center, Paris, France \\ *Both authors contributed equally to this work \\ Correspondence to: Yorick Gitton, email: yorick.gitton@inserm.fr
}

Keywords: FOXP2 factor, oncogene, cancer, prognosis, language

Received: March 01, $2018 \quad$ Accepted: April 02, $2018 \quad$ Published: April 18, 2018

Copyright: Herrero and Gitton et al. This is an open-access article distributed under the terms of the Creative Commons Attribution License 3.0 (CC BY 3.0), which permits unrestricted use, distribution, and reproduction in any medium, provided the original author and source are credited.

\section{ABSTRACT}

FOXP2 encodes a transcription factor involved in speech and language acquisition. Growing evidence now suggests that dysregulated FOXP2 activity may also be instrumental in human oncogenesis, along the lines of other cardinal developmental transcription factors such as DLX5 and DLX6 [1-4].

Several FOXP family members are directly involved during cancer initiation, maintenance and progression in the adult [5-8]. This may comprise either a prooncogenic activity or a deficient tumor-suppressor role, depending upon cell types and associated signaling pathways. While FOXP2 is expressed in numerous cell types, its expression has been found to be down-regulated in breast cancer [9], hepatocellular carcinoma [8] and gastric cancer biopsies [10]. Conversely, overexpressed FOXP2 has been reported in multiple myelomas, MGUS (Monoclonal Gammopathy of Undetermined Significance), several subtypes of lymphomas [5,11], as well as in neuroblastomas [12] and ERG fusion-negative prostate cancers [13]. According to functional evidences reported in breast cancer [9] and survey of recent transcriptomic and proteomic analyses of different tumor biopsies, we postulate that FOXP2 dysregulation may play a main role throughout cancer initiation and progression. In some cancer conditions, FOXP2 levels are now considered as a critical diagnostic marker of neoplastic cells, and in many situations, they even bear strong prognostic value [5]. Whether FOXP2 may further become a therapeutic target is an actively explored lead. Knowledge reviewed here may help improve our understanding of FOXP2 roles during oncogenesis and provide cues for diagnostic, prognostic and therapeutic analyses.

\section{INTRODUCTION}

FOXP2 belongs to the extensive family of more than forty-three Forkhead box-winged helix transcription factors organized into nineteen sub-families. They are endowed with both activating and, more often, repressing transcriptional activities [14]. Conserved FOXP2 expression is detected throughout several developing tissues, including the brain [15] [16] [17]. This organ has been a major focus of FOXP2 research as a gene involved in language and speech acquisition in modern humans, vocalizations in mouse and other communicative skills [18].

Clinically, chromosomal lesions involving the
FOXP2 locus are associated with impaired brain development and neuronal differentiation, and give origin to complex neural disorders - the most salient impacting language processing and speech [19]. Genetic invalidation of the murine Foxp2 leads to severe developmental delays, motor defects, absence of ultrasonic sounds that juveniles emit when separated from their mothers, and premature death [20]. However, FOXP2 is also expressed in a large spectrum of other embryonic, postnatal and adult tissues, where its dysregulation has been observed to be associated with cancer conditions. The present manuscript reviews the features of FOXP2 genomic context, its transcripts, its protein isoforms and targets, and data that substantiate the notion of a critical contribution of FOXP2 to cellular 
barriers against cancer progression. Such information provides an integrated view of available evidence which may allow to append "oncogene" to its "language" gene designation.

\section{Structural characterization of the transcription factor-encoding $F O X P 2$ gene}

\subsection{DNA}

\subsubsection{Genomic landscape of $F O X P 2$ : a tumorigenic hotspot?}

FOXP2 has been designated previously as follows: SPCH1 ("speech and language disorder 1"), TNRC10 ("trinucleotide repeat containing 10"), CAGH44 ("CAG repeat protein 44"), and DKFZp686H1726 (HNGC nomenclature). The genomic context of human FOXP2 is the large arm of the chromosome 7, on the forward strand, from Ensembl coordinates chr7:114,086,327 to $114,693,772$ in GRCh38:CM000669.2 (Jun. 2017; Ensembl) (Figure 1). Previously UCSC had located it in chr7:114,414,997-114,693,768 in GRCh38/hg38 (Dec. 2013; UCSC).

This locus abuts the junction of Giemsa-negative and positive bands q31 and q32. Noticeably, these bands are prone to somatic chromosomal instability and subsequent rearrangements, favoring tumorigenesis and cancer progression $[21,22]$. Furthermore this region has been reported to harbor fragile genomic hotspots impacting neighboring genes [23], as depicted in the bottom part of Figure 1. In the vicinity of the FOXP2 locus, within $5 \mathrm{Mb}$, neighboring protein-encoding genes include PPP1R3A, GPR85, BMT2, TMEM168, LSEM1, IFRD1 centromerically; and MDFIC, TFEC, TES, CAV2, CAV1 and $M E T$, telomerically. Among these genes, TES/TESTIN and $M E T$ are of particular oncogenic interest through their role as a $7 \mathrm{q} 31$ hotspots reported for genomic instability leading to invalidation of these tumor-suppressor $(\underline{T E S})$ and pro-oncogenic $(\underline{M E T})$ factors [24,25].

Three farther hotspot candidate genes have been involved in a variety of pathological conditions when deficient, including neoplasia:

i) The SMO (SMOOTHENED gene), at 7q32, a frizzled-class receptor belonging to the SONIC HEDGEHOG (SHH) pathway, associated with oncogenic conditions including basal cell carcinoma, malignant glioma, medulloblastoma, leukemia, and cancers of the breast, lung, pancreas, and prostate [26].

ii) the $B-R A F$ proto-oncogene at $7 \mathrm{q} 34$, a serine/ threonine kinase associated with leukemia, melanoma, thyroid, ovarian, colon and lung cancer [27].

iii) the EZH2 (ENHANCER OF ZESTE 2) polycomb repressive complex 2 subunit, at 7q36, a transcriptional co-repressor, frequently overexpressed in many cancer types, including prostate, breast, bladder, ovarian, lung, liver, brain, kidney, gastric, oesophageal, and pancreatic cancers and melanoma [28].

Whether these oncogenic situations result from or depend upon isolated or combined defects of FOXP2 and its neighbors remains unclear. We have collated observations from cancer databases on genomic lesions involving genes in the vicinity of FOXP2 in section 5.2 (see further).

\subsubsection{Epigenomic landscape of $F O X P 2$}

\subsubsection{Contradictory evidence for imprinting of the FOXP2 locus}

Dysregulation of parental allele-specific expression of genes, resulting from altered imprinting marks

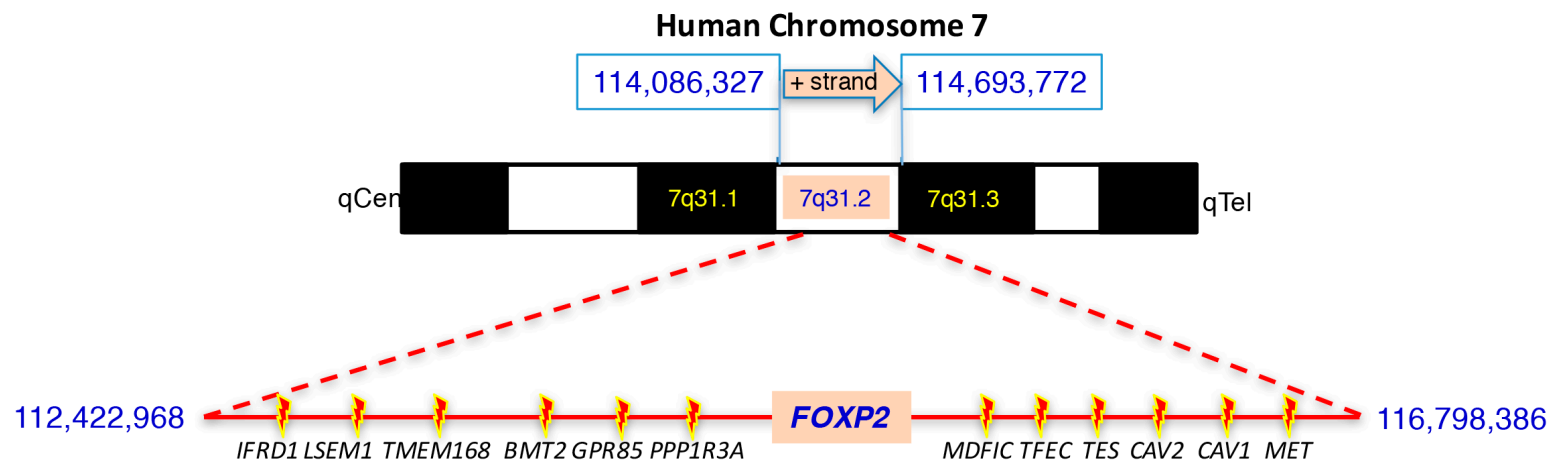

\section{1: Fragile genomic hotspots}

Figure 1: DNA. HUMAN FOXP2 LOCUS (NCBI ID: 93986; Atlas ID: 40633). Location of the FOXP2 locus on the long arm of chromosome 7 band 7q31.1 (fragment, ENSEMBL coordinates GRCh38:CM000669.2). FOXP2 is located within a 5 Mb-large region of fragile genomic hotspots involving the highlighted neighboring genes. 
deposited onto specific loci, has often been associated with altered copy number variation and early stages of oncogenic processes. Loss of imprinting in both human and mice, impacting either global genomic territories or more specific loci, is a precious diagnostic hallmark for the earliest events of malign transformation - and the most frequent defect during tumorigenesis. To assess the susceptibility of the FOXP2 locus to genomic lesions which may have deleterious oncogenic consequences, we first reviewed the status of the imprinting of this locus.

The FOXP2 locus is subject to conflicting interpretations with regards to the imprinting status. A first series of parental origin of polymorphisms expressed in a cohort of patients has uncovered a bias towards a maternal imprinting leading to an exclusive paternal expression of FOXP2 [29]. However, other authors have revisited this issue using more sensitive methods consisting in decoding the parental origin of the FOXP2 transcripts [30]. Their observations were consistent with a biallelic expression of FOXP2, ruling out any role for imprinting. So far, direct bisulfite sequencing, which would bring more sensitive evidence for differential imprints on the FOXP2 locus, has not been reported - although efforts to gain such insight are appearing as in the MethCNA database [31]. In contrast, a neighboring region on 7q31 (GRM8) is imprinted [32]. Alternatively, an indirect imprinting mechanism was uncovered which influences the parental allelic expression of FOXP2 in the immune cell lineage of one individual afflicted by verbal dyspraxia [33]. A regulatory region as far as $3 \mathrm{Mb}$ upstream of FOXP2 harbors a putative regulatory element that controls FOXP2 expression level and is subjected to parental imprinting. Whether this mosaic process concerns neurons involved in language remains to be determined. Along the same line of analysis, the rare case reports involving loss of heterozygosity of FOXP2 have so far been associated with neuro-developmental disorders but not with cancer [34].

\subsubsection{The FOXP2 locus, a target cluster for cancer- associated epigenomic conversions}

In cancer, loss of expression of genes occurs frequently by hypermethylation of promoter $C p G$ islands. Transcriptomic analysis of twelve genes within the $4.12 \mathrm{Mb}$ region centered around FOXP2 (Figure 1) in primary prostate cancer cells has uncovered a common trend towards severe down-regulation of this set of genes [35]. This phenotype is associated with H3K27me3 hyper-methylation of $C p G$ islands in promoters of this domain, with a concomitant de-acetylation, generalized to the whole domain. Clinical samples displayed identical cancer-associated epigenetic changes of these clustered genes. FOXP2 thus belongs to a domain featuring cancer epigenome consolidation, priming clustered genes for generalized dysregulation through alteration of chromatin accessibility to transcription. The mechanism linking this transformation to a growth advantage in pre-oncogenic cells remains to be elucidated. Consistently, some of the genes in this cluster have roles in neoplasia, including $M E T$ (see above) whose hypomethylation and acetylation have been associated with its high expression in some cancers [24]; as well as FOXP2 (detailed below in section $5)$.

\subsubsection{Cis-regulatory control of FOXP2: a target for cancer signaling cascades?}

\subsubsection{FOXP2 Promoters}

Somatic epigenetic lesions contribute to major disruptive processes involved in the transition from preoncogenic to oncogenic conditions. Some of these events are of critical importance in normal cellular physiology as hallmarks for regulation of the transcription of associated loci. Both upstream and downstream regulatory elements have been identified as impacting FOXP2 expression [36].

Combining data mined from Ensembl and Genomatix (version 3.9), we have identified thirteen alternative promoters which have been experimentally validated as controlling the transcription of the twentyseven isoforms from the FOXP2 locus:

- Seven of these promoters are centromeric to the transcription start site of the longest and predominant product (FOXP2-201, ENST00000350908.8, NM_014491; Figure 2). This product was previously designated as FOXP2_001.

- Two promoters telomeric to the most downstream transcript were identified and functionally validated [37].

- Three promoters are intronic (Introns 1, 4, 13),

- One promoter is exonic (Exon 1).

In human tissues four alternative promoters have been validated in vitro - those within exons $\mathrm{S} 1$ and exon 2 (Figure 2) being responsible for ubiquitous and basal distribution of FOXP2 transcripts [38]. It remains to be deciphered why three of these promoters engage FOXP2 production from the same transcriptional start site.

The complexity of this configuration suggests multiple levels of control of FOXP2, which may be relevant to oncogenic processes. In the next paragraph, we focus upon the promoter which controls the most abundant form of FOXP2 transcripts, emphasizing oncogenic signaling. This does not rule out an oncogenic role for the other promoters and associated RNA isoforms.

\subsubsection{Regulatory elements associated with FOXP2 expression}

Centromeric regulatory elements place FOXP2 under cancer-prone signaling cascades. Using Genomatix, we screened for the strongest consensus binding sites of different transcription factors mediating oncogenic signaling. We researched only one (Genomatix ID\# GXP_94278, position 7:114,413,997-114,415,423; $1427 \mathrm{bp}$ ) of the thirteen experimentally validated promoters 
as conditioning the production of the predominant transcript (FOXP2_201) found in most cell types. We refer the reader to these types of analytic tools to examine whether other, less expressed, transcripts might fall under the control of the alternative promoters. This GXP 94278 promoter has been experimentally validated and harbors candidate binding sites for effector transcription factors of diverse oncogenic signaling pathways, from which we selected TP53 and LEF-1:

- TP53: TP53 is a ubiquitous tumor-suppressor gene reported to be mutated in half of human cancers. The encoded factor P53 exerts its protective roles through a series of effectors, which broadly prevent excessive proliferation by dampening upon cell cycle progression and by inducing growth arrest in overproliferating cells undergoing a neoplastic transformation by triggering apoptosis. The main activating event of P53 is DNA damage associated with oncogenic initiation. We found a bona fide binding site for P53 spanning promoter GXP_94278, positions 681-705 with a taagCAAAcccaagacaagcatttc sequence (with the core in capitals). Another position lies at chr7: 114,060,411$114,060,421$ - (TAGGCAGGTCT), which is identified by the QIAGEN promoter analysis tool as a P53 binding site in human cells, among 200 other transcription factors susceptible to bind the upstream territory of the FOXP2 locus. These elements are compatible with the notion that FOXP2 expression status may be a direct target of TP53 activity.

- LEF1 (Lymphoid Enhancer Binding Factor 1):
We emphasized our analysis on this factor because it is the downstream effector of WNT signaling in numerous normal and pathological conditions - including during oncogenesis. In the FoxP2 genomic region at least six Lef1 binding sites were common between zebrafish, mouse and humans [39]. One study in zebrafish found a promoter controlling FoxP2 expression and detected a matching orthologous sequence in the human genome [40]. They identified the sequence (zebrafish: ttgtgggctGCTTTCATCtgtgggttaa; orthologous to human: atgatcagtGCTTTCATCtttattttaa) at $-8.5 \mathrm{kbp}$ upstream from human FOXP2_201 transcription start site. BLASTing this sequence with a more recent genome version locates its exact $17 \mathrm{bp}$ match within the first FOXP2 intron, at position 7: 114,417,683-114,417,710. Furthermore, the BLAST returns a perfect match on another chromosome at position 12: 90,502,826-90,502,988 - but without any obvious gene encoding sequence. The possibility remains open that it acts as a long-range cis-regulator. To further our understanding of a putative LEF1 regulation of FOXP2 expression we screened the upstream GXP_94278 promoter (see above). We found a set of twelve binding sites with high scores, including a stretch of two on the plus strand, separated by $14 \mathrm{bp}$ - in which a minus strand site is also located. While speculative, this configuration opens the possibility for a putative LEF1-dependent regulation of $F O X P 2$ transcription associated with human oncogenesis. Such regulation could be independent or initiated by WNT signaling [41], in a cascade which may involve a $\mathrm{WNT} \rightarrow \mathrm{LEF} 1 \rightarrow$ FOXP2 activation sequence.

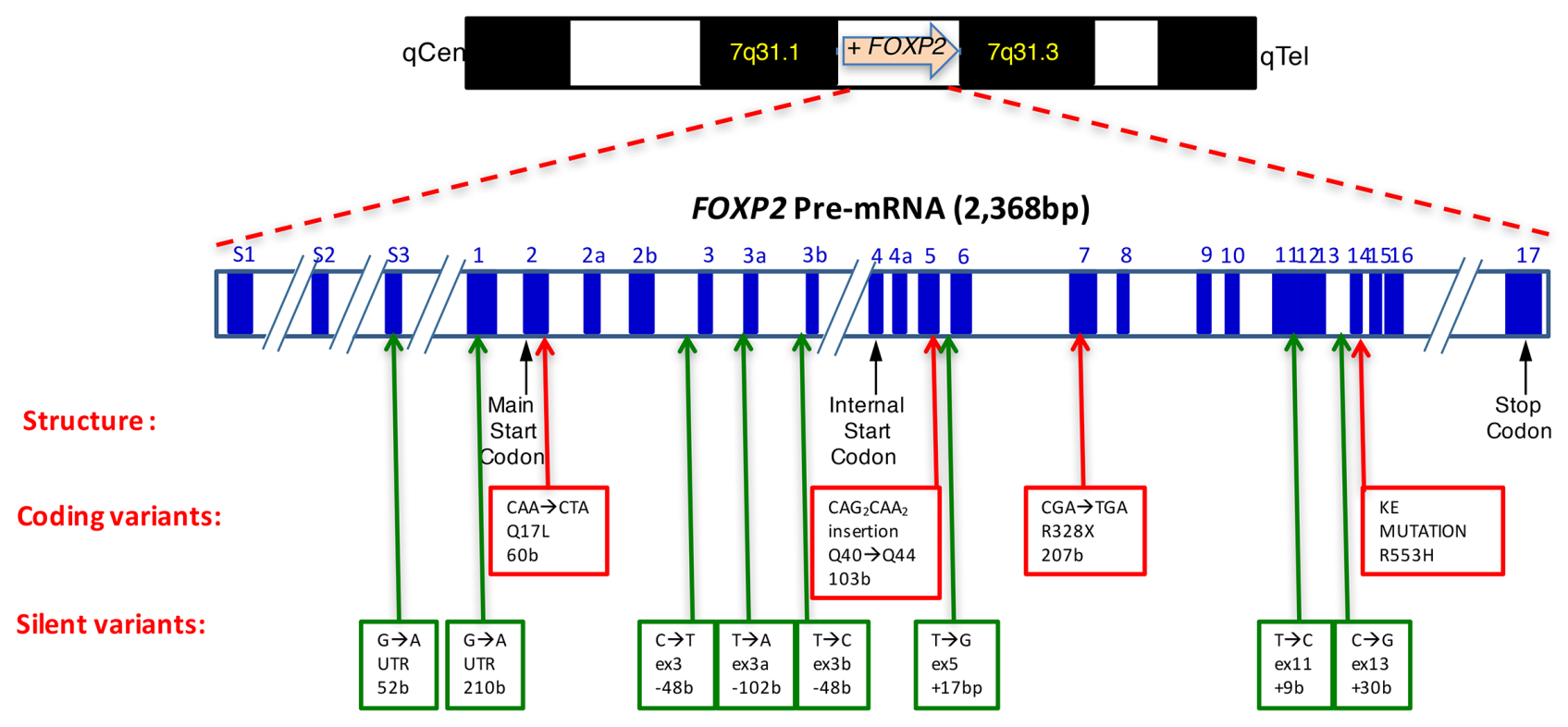

Figure 2: RNA. HUMAN FOXP2 pre-mRNA structure. It encodes for 17 exons (blue segments), with exon 1 being the first in the predominant FOXP2 isoform. Bottom part: location of mutational variants assembled from different sources [19,131,176,177], including DECIPHER database. 
Further analyzing the possibility of oncogenic factor binding to FOXP2 regulatory elements may yield new cues relevant to oncogenesis. We refer the reader to recent studies describing FOXP2 involvement during oncogenic processes for other major oncogenes, including MYC [9].

A recent genomic analysis reports the presence of two additional regulatory elements with enhancer function in the telomeric territory separating FOXP2 from its neighbor, $M D F 1 C$ [37]. These enhancers have been observed to be disrupted in a child with language and speech disorder. The requirement for these two elements in driving proper FOXP2 expression levels was functionally validated in human cell lines [37]. These data lend support to the hypothesis that FOXP2 expression falls under a large array of regulatory elements, which may increase the probability of dysregulation during oncogenic processes.

\subsubsection{A $F O X P 2$ intragenic regulatory element: an oncogenic target?}

The FOXP2 locus hosts many sequences which act as hallmarks of insulating regions [42]. Browsing the regulatory build channel of ENSEMBL for human FOXP2 shows that at least ten CTCF regulatory binding sites are distributed across numerous introns, and also outside of the locus. These 'CTCF' regions are expected to insulate $F O X P 2$ intragenic regulatory elements from acting over long distance loci [43]. Hence, regulatory elements located within $F O X P 2$ would be expected to locally impact $F O X P 2$ transcription. In $H$. sapiens FOXP2 intron 8, a single nucleotide substitution at position Hsa 7:114,076,877 was identified, which was not present in the $H$. neanderthalensis genome [44]. The modern allele favored the binding of the neuronal-specific transcription factor POU3F2/OCT3, which promoted FOXP2 transcription - in contrast to the ancestral allele, where POU3F2/OCT3 binding was inefficient. The authors characterized this site as a putative internal regulatory element dedicated to enhancing neuronal expression of FOXP2 under the control of POU3F2/OCT3. While the role of this evolutionary modification in language acquisition remains to be completely elucidated, the fact that it involves POU3F2/OCT3 activity bears some relevance with brain oncogenesis. Indeed, POU3F2/OCT3 overexpression has been correlated with neuroblastoma and glioblastoma in both human brain and neuroblastomaderived cell lines (SH-SY5Y) $[12,45,46]$.

\subsection{RNA}

\subsubsection{Transcripts}

\subsubsection{Description}

Transcription from $F O X P 2$ yields a 2,368bp long pre-messenger RNA (from 114,415,055 to 114,690,100) (Figure 2). It harbors twenty-seven splice variants - four transcripts being untranslated. The nineteen coding ones range from $625 \mathrm{~b}$ to $8300 \mathrm{~b}$ due to alternative splicing sites throughout the precursor transcript. The prevalent human isoform encodes for a 715AA protein from 17 exons (Isoform FOXP2-201, ENST00000350908.8, CCDS 43635.1, NM_014491, Uniprot O15409), which have been detailed elsewhere [38]. Furthermore, four antisense non-coding transcripts have been characterized. The composition of seventeen transcript variants is detailed and updated on a dedicated NCBI page, and available from the human FOXP2 Ensembl page. Genomic comparisons have shown that FOXP2 is the gene harboring the most ultraconserved sequences in its introns, suggesting a wide array of putative regulatory elements. $F O X P 2$ has fusion genes with COG5 (7q22.3), RCF3 (13q13.3), RPL36 (19p13.3) and SFTPB (2p11.2).

\subsubsection{Exception}

FOXP2 sequence analysis of one familial mutation $(\mathrm{R} 553 \mathrm{H})$ [19] has led to the discovery of a longer transcript endowing FOXP2 with an unusual poly-glutamine/polyproline stretch, forty $\mathrm{CAG} / \mathrm{CCG}$ repeats long, that has been found to be aggregated when ectopically expressed in COS cells [47].

One of the functional consequences of these expansions is the initiation of a cellular stress signaling cascade. Mechanistically, FOXP2 is a binding partner for the nuclear translocation of POT1 (Protection of Telomeres I). FOXP2 promotes the nuclear translocation of POT1, but the mutated FOXP2(R553H) protein related to speech-language disorder, partially prevents it $[48,49]$. This may account for the altered distribution and function of this FOXP2 mutant form which cannot exert its nuclear functions.

\subsubsection{Post-transcriptional regulation of FOXP2 mRNA}

FOXP2 mRNAs are numerous and subjected to intensive splicing. While this property may in itself constitute a mode of regulation of FOXP2 expression we have focused here on microRNA-mediated regulation of $F O X P 2$. We have surveyed both predicted and validated miRs and identified a set of FOXP2-targeting microRNAs from the following databases: targetscan 7.1, mirBase 21, $\underline{\text { mirdb}}$. Close to $284 \mathrm{miRs}$ are predicted to target human FOXP2, including 44 with a score above $95 \%$.

Of particular clinical interest is miR-3666 (NR_037439), a mirtron located within the FOXP2 locus at $7[+]: 114653345-114653455$ (GRCh 38) in intron 9. This intronic transcript is spliced out from all FOXP2 pre-mRNAs isoforms. While not confidently annotated, the Targetscan database proposes its analysis with regards to a significantly close family of miRs: miR$130-3 \mathrm{p} / 301-3 \mathrm{p} / 454-3 \mathrm{p}$. Such a co-expression scheme raises several issues. First, both miR-3666 and FOXP2 play a larger regulatory role than expected by examining the sole impact of FOXP2 on transcription: putatively 
downregulated targets of miR-3666 may complexify the impact of FOXP2. In particular, repressive role of FOXP2 on target genes may be due in part to miR-3666 and begs for a thorough analysis of the direct binding of FOXP2. Next, alternative start sites of transcription combined with alternative splicing might bring an unexpected level of complexity in the balance between the host gene and its encoded microRNA. Lastly, mutations in the FOXP2 locus might be consequential for the phenotype whether each or only one of these elements are concerned. Recently, computational evidence has been proposed for an auto-repression of FOXP 2 by miR-3666 in cell lines, and uncovered a set of several hundred genes conjointly targeted by both, with a bias toward genes putatively involved in schizophrenia and/or autism spectrum disorder [50]. In the context of cancer progression, miR-3666 has been associated with several cancer types and displayed reduced transcripts levels in lung [51], thyroid [52] and pituitary cancer cells [53]. However, how defective FOXP2 expression associated with miR3666 misexpression might promote oncogenic initiation, maintenance, or aggressiveness remains to be assessed.

Additionally, other microRNAs might be associated with dysregulation of FOXP2 expression levels in oncogenic conditions. One frequent candidate is the miR190. Of its four isoforms (a, a-3p, a-5p, b), miR-190a has been experimentally validated and located on the plus strand of chromosome 15, within the second intron of the gene TALIN2 (TLN2). The salient feature of this host gene is that it has been demonstrated to be down-regulated in cancer cell lines and biopsies. We discuss the functional consequences of this observation in sections 'entity' devoted to the hepatocellular carcinoma [54], breast cancer [55] and gastric cancer [10].

Conversely, up-regulated miR-190 has been reported in a variety of conditions such as bladder cancer, breast cancer, lung cancer, liver cancer, and colorectal cancer, as well as in bronchial epithelial cell cancerization induced by arsenic [56-61]. Noticeably, in glioblastomas and osteosarcomas in in vivo models of human cancer in immune-compromised mice, up-regulation of miR-190 led to prolonged tumor dormancy [55]. Altogether these observations in diverse oncogenic conditions point to the susceptibility of FOXP2 expression levels to the activity of miR-190, which in some cases has been associated with oncogenic initiation.

In another large scale oncogenic study [9], FOXP2 expression level has been shown to directly depend upon the activity of a coordinated set of microRNAs. The authors observed that the malignancy of breast cancer cells (BCCs) was enhanced upon exposure to incoming mesenchymal stem cells populating the breast tumor stroma. This interaction triggers the activation of a TWIST-dependent signaling cascade, which has two mechanistic consequences (Figure 6). First, it activates a set of adhesion-related genes in BCCs. Second, TWIST activates two waves of miRs: the 199a-214 cluster and a set of four other microRNAs (miR-762, miR-1915, let-7b, and miR-34a). All these miRs share FOXP2 as a validated target, leading to the down-regulation of this gene. Subsequently, this cascade converged on and repressed the expression of FOXP2 promoting cancer stem cell (CSC) and metastatic traits. This condition correlated with poor survival in breast cancer.

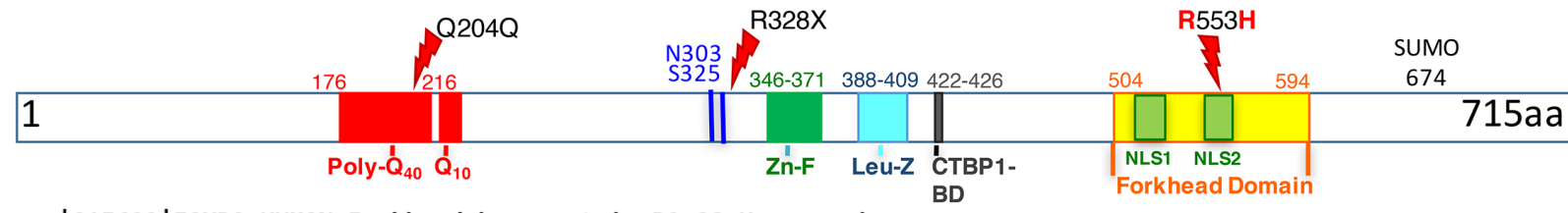

>sp|015409|FOXP2_HUMAN Forkhead box protein P2 OS=Homo sapiens MMQESATETISNSSMNQNGMSTLSSQLDAGSRDGRSSGDTSSEVSTVELLHLQQQQALQA ARQLLLQQOTSGLKSPKSSDKORPLQVPVSVAMMTPQVITPQOMOQILQQQVLSPPQQLQA LLOQOOAVMLOOOOLOEFYKKOOEOLHLQLLLO000000000000000000000000000C

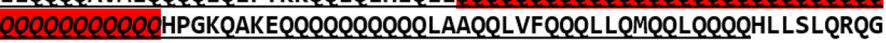
LISIPPGQAALPVQSLPQAGLSPAEIQQLWKEVTGVHSMEDNGIKHGGLDLTTNNSSSTT SSNTSKASPPITHHSIVNGQSSVLSARRDSSSHEETGASHTLYGHGVCKWPGCESICEDF GQFLKHLNNEHALDDRSTAQCRVQMQVVQQLEIQLSKERERLQAMMTHLHMRPSEPKPSP KPLNLVSSVTMSKNMLETSPQSLPQTPTTPTAPVTPITQGPSVITPASVPNVGAIRRRHS DKYNIPMSSEIAPNYEFYKNADVRPPFTYATLIRQAIMESSDRQLTLNEIYSWFTRTFAY FRRNAATWKNAV R HNLSLHKCFVRVENVKGAVWTVDEVEYQKRRSQKITGSPTLVKNIP. SLGYGAALNASLQAALAESSLPLLSNPGLINNASSGLLQAVHEDLNGSLDHIDSNGNSSP GCSPQPHIHSIHVKEEPVIAEDEDCPMSLVTTANHSPELEDDREIEEEPLSEDLE
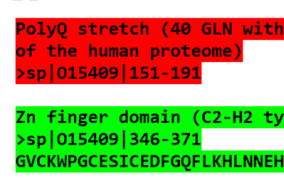

Leucine Zipper domain : >sp $|015409| 388-409$ VQQLEIQLSKERERLQAMMTHL

CTBP1-binding domain DNA Binding domain (Forkhead type) : >sp|015409|504-594

RPPFTYATLIRQAIMESSDRQLTLNEIYSWFTRTFAYFRRNAATWKNAVRHNLSLHKCFV RVENVKGAVWTVDEVEYQKRRSQKITGSPTL

Functional SNP (R553H, natural variant rs121908377 in human family with speech disorder )

$>$ sp $|015409| 553-553$

Figure 3: PROTEIN. Human FOXP2 main protein isoform (Uniprot O15409). Structural and functional domains are highlighted. The Forkhead P2 domain harbors two nuclear localization signals ('NLS') [70]. Two major mutated variants are indicated above, with R328X interrupting the protein and $\mathrm{R} 553 \mathrm{H}$ altering its subcellular localization (KE family verbal dyspraxia mutation). The two human lineagespecific aminoacids N303 and S325 are indicated in blue. The sumoylation site (K674) is indicated. A Q204Q substitution observed in multiple cancers is discussed in Figure 5. 


\subsection{Protein}

\subsubsection{Biochemical properties}

A 715-aa transcription factor (Uniprot \#O15409) is encoded by the main FOXP2 mRNA isoform FOXP2_201 (Figure 2). It features five main functional domains illustrated by Figure 3. The molecular mass nears $80 \mathrm{kDa}$ and its DNA binding activity requires dimerization. The 100-aa Forkhead domain (or 'winged-helix') is located in the C-terminal part and accounts for FOXP2 DNA binding activity, as has been detailed upon determination of the crystal structure of its interaction with DNA [62]. A detailed comparison of the protein structures across families of Forkhead factors has been reviewed elsewhere [63]. Biostructural analysis has identified a FOXP2 consensus binding sequence as 5'-CAAATT-3' [62]. Furthermore, swapping events involving the $\mathrm{N}$-terminal domain adapt FOXP2 interactions with other monomers, including with its paralogs FOXP1 and FOXP4, which may provide functional plasticity to FOXP2 activity depending upon the cellular context [64]. A recent structural dissection of FOXP2 DNA binding has identified three rate and affinity modalities, respectively enabling fast genome browsing, medium target site detection and strong binding to best affinity sites engaging FOXP2 into transcriptional activity [65]. The zinc finger and leucine zipper domains have been hypothesized to be involved in these interactions $[65,66]$.

Furthermore, FOXP2 harbors a binding domain for the co-repressor CtBP1 (C-Terminal Binding Protein-1, Figure 3) which has been experimentally validated $[34,66]$ and may be involved as a tumor suppressor in oncogenic processes through interaction with the BRCA1/2 breast cancer oncofactors $[67,68]$.

FOXP2 may also behave as a chromatin bookmarking agent since it has been reported to associate with the NuRD chromatin remodelling complex which, furthermore, harbors HDAC components responsible for the repressing activity of FOXP2 [69].

Another structural feature of FOXP2 is the presence of two nuclear localization signals (NLS) embedded within the Forkhead domain. These may force FOXP2 translocation from the cytoplasm to the nucleus as demonstrated by cellular mutagenesis assays [70]. Interestingly, the R553H mutation of the KE family (see Figures 2 and 3, and pathology section 4 below) has been demonstrated to hinder FOXP2 nuclear localization due to its invalidation of the C-terminal NLS [70].

Finally, the N-terminus of FOXP2 displays the longest poly-glutamine tract of all known proteins - a distinct feature of protein misfolding disorders impacting the nervous system [34].

The FOXP2 protein undergoes a major posttranslational modification by SUMOylation (Small Ubiquitin-like Modifier). It has been demonstrated to be a critical regulatory mechanism of FOXP2 activity [71]. Interaction between FOXP2 and PIAS3, a critical component of this modification, results in the addition of a SUMO group to FOXP2 at the highly conserved position K674 (Figure 3) by PIAS3 [72]. This leads to the massive redistribution of FOXP2 into nuclear speckles of granular heterochromatin [34]. However, the functional importance of this modification remains to be clarified since PIAS3 invalidation does not prevent FOXP2 transcriptional repressive activity in some cells (Hela and HEK293), [71] but does impact it in other cells (MCF7 and SH-SY5Y, but also HEK293) [72]. Noticeably, the SUMOylation of the murine Foxp2 had been previously hypothesized to promote the dissociation of the Foxp2 dimer from its target promoter, leading to an overall reduction of Foxp2 transcription suppressive activity [74].

\subsubsection{Functional features}

FOXP2 has been demonstrated to exert both promoting and, more often, repressing activities of the transcription of target genes. This suppressive activity might be accounted for by the Zinc finger domain [69]. Combination of chromatin immunoprecipitation with microarray analysis in cells dissected from fetal human brains has identified a strong consensus for FOXP2 response element in target loci [75]. Interestingly, it has been observed that the human ortholog displays a unique substitution at position 303 of this transcriptional repressor domain ( $\mathrm{Thr} \rightarrow \mathrm{Asn}$ ), which sets it apart from all other species [76]. Whether this modification bears functional relevance remains to be determined.

A survey of directly bound loci yielded candidate FOXP2-binding sequences with a core underlined within CAAATT as the most probable target, even if some other alternative sites were also reported $[62,75,77,78]$. Collectively, most studies unveil a predominantly repressor role for FOXP2 upon transcription of target genes, even if some exceptions report activating properties [17,75,78-82].

One major functional aspect of FOXP2 activity stems from its dimerization with its paralogs FOXP1 and FOXP4. Indeed, different combinations of FOXP1/2/4 dimerization severely affect gene expression [64]. This property may have oncogenic consequences. FOXP1 and FOXP2 have been observed to be widely co-expressed in specific territories [83] and to co-operate at least during development [84]. Deficiency of both factors has been involved in malformative processes leading to autistic, language and cognitive deficiencies, among others [85]. Additionally, dysregulated FOXP1 expression has been associated to deleterious oncogenic activities, including hepatocellular carcinoma, breast, renal, prostate and endometrial cancer [86-93]. Similarly, FOXP4 has been shown to interact with FOXP2 in several developmental processes, such as neuronal differentiation and migration [94]. FOXP4 expression was aberrant in several breast 
cancer cell lines and kidney cancer $[95,96]$. However, studies on FOXP1/2/4 interaction in oncogenesis are lacking. Considering that tissue-specific combination of FOXP2 homodimers and heterodimers may modulate the transcription of specific target genes, it appears crucial to better understand a putative synergy of FOXP2 with its paralogs in oncogenesis.

Proteome-wide surveys report experiments showing the physiological interaction of FOXP2 with 29 other factors (NIH-gene FOXP2 page) [14]. Deciphering which of these partners, such as FOXP1, CtBP1, MAPK3, GATAD2B, might be involved in oncogenic process along with FOXP2 should improve our understanding of gene networks underlying cancer progression.

\section{Distribution of FOXP2 gene expression products}

\subsection{Central nervous system}

Transcriptomic analyses have identified Foxp2 transcripts in mouse cortical neurons mainly, but also in astrocytes and oligodendrocytes, as well as endothelial cells [97]. Foxp2 is expressed in the neocortex, the striatum, the amygdala, the thalamus, the hypothalamus, the hippocampus and the cerebellum $[15,16,98,99]$. In the developing human hindbrain, FOXP2 protein has been also detected at strong levels within brainstem nuclei and spinal cord [100]. Brain FOXP2 expression broadly covers territories responsible for language acquisition and production, including speech-associated motor control, in particular in the developing basal ganglia. The functional consequence of this expression is discussed in the section "4.1. FOXP2 and language".

FOXP2 expression in human brain is elevated during mid-gestation [100], declining postnatally to nearly undetectable levels in adult, while in rodent and zebra finch brain Foxp2 remains at high levels from neurogenesis through adulthood (Human Brain Transcriptome database) [101]. According to the Human Protein Atlas, femalespecific tissues carry more expression than male ones, while in mice Foxp2 protein is significantly higher in multiple regions of the developing male brain compared with females [102].

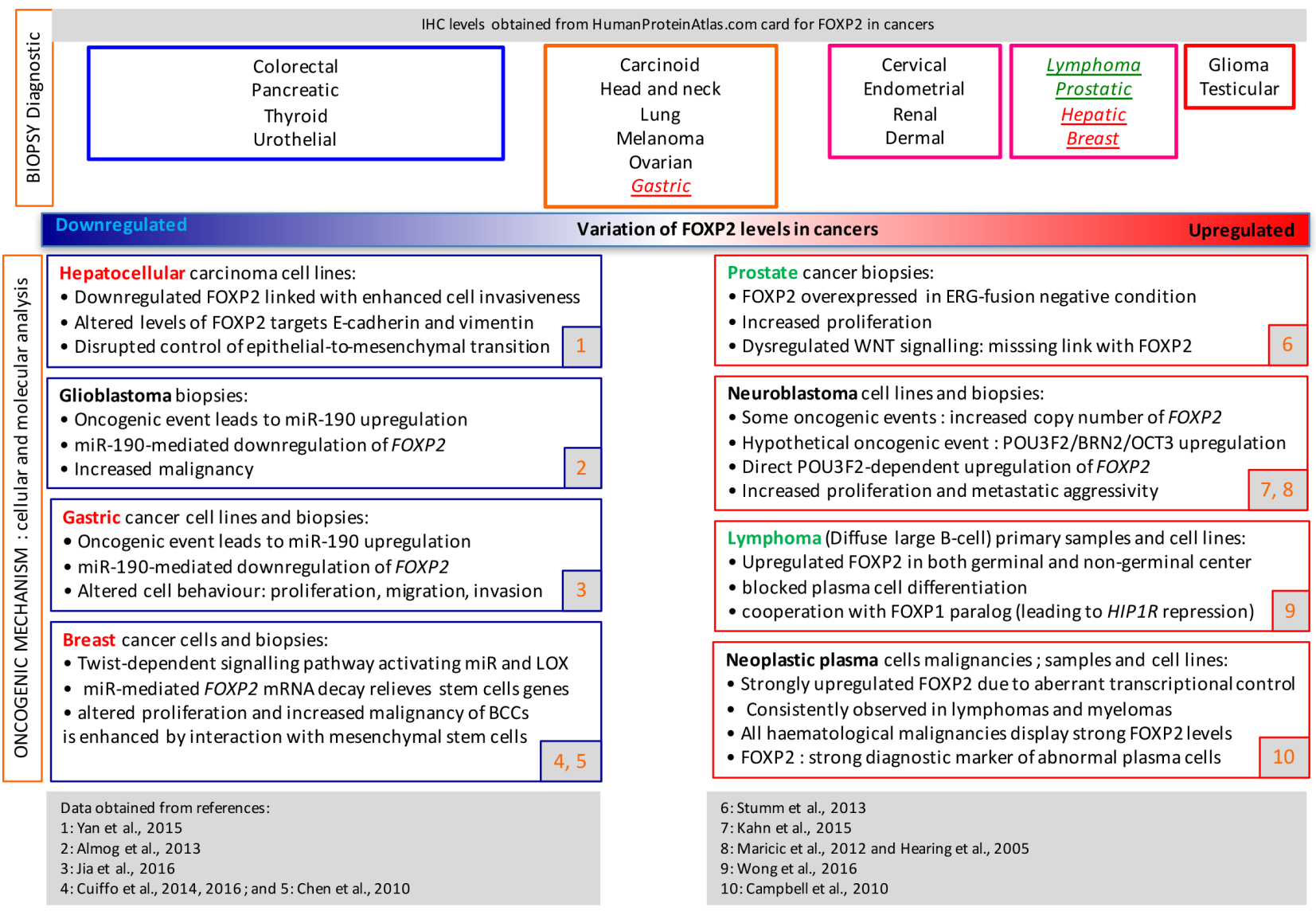

Figure 4: Onco-diagnostic relevance of FOXP2 expression level, comparing immunophenotyping in cancer biopsies (top pannel) with mechanistic data (bottom pannel). Compared regulations: red writing= different results; green writing=identical results. 


\subsection{Outside CNS in tissues concerned with tumorigenesis}

On a system-wide level, besides the brain, FOXP2 is mainly detected in endocrine (thymus), muscular, cardiac, vascular, pulpmonary, gastrointestinal and urogenital tissues [66,84]. FOXP2 displays expression in 53 human tissues surveyed - the highest levels being detected in gastro-intestinal and urogenital systems (see UCSC Gencode GTEx, J. Kent and B. Raney).

\section{Functional properties of FOXP2 in normal conditions}

\subsection{Subcellular localization of FOXP2}

Immuno-colocalization and functional assays have identified a predominant distribution for FOXP2 within the nuclear compartment of the cell. Mutated forms, including the $\mathrm{R} 553 \mathrm{H}$ and $\mathrm{R} 328 \mathrm{X}$ clinical alleles, have been reported to segregate FOXP2 in the cytoplasm and to preclude their transcriptional activity, due to DNA-binding failure [49]. This misallocation has been hypothesized to induce cellular stress as endoplasmic reticulum stress markers were observed to accumulate in these conditions [103]. As previously indicated, two nuclear localization signals enforce FOXP2 nuclear translocation, with SUMOylation further segregating it in the active heterochromatin. Noticeably, a rare natural isoform of Foxp2 without Forkhead domain has been reported in the mitochondrial compartment of murine cerebellar Purkinje cells [49].

\subsection{FOXP2 dimerization}

FOXP2 interacts with its paralogs FOXP1/3/4 through heterotypic dimerization, an important feature of its transcriptional activity. Additionally, numerous data have shown that FOXP2 functions may further depend

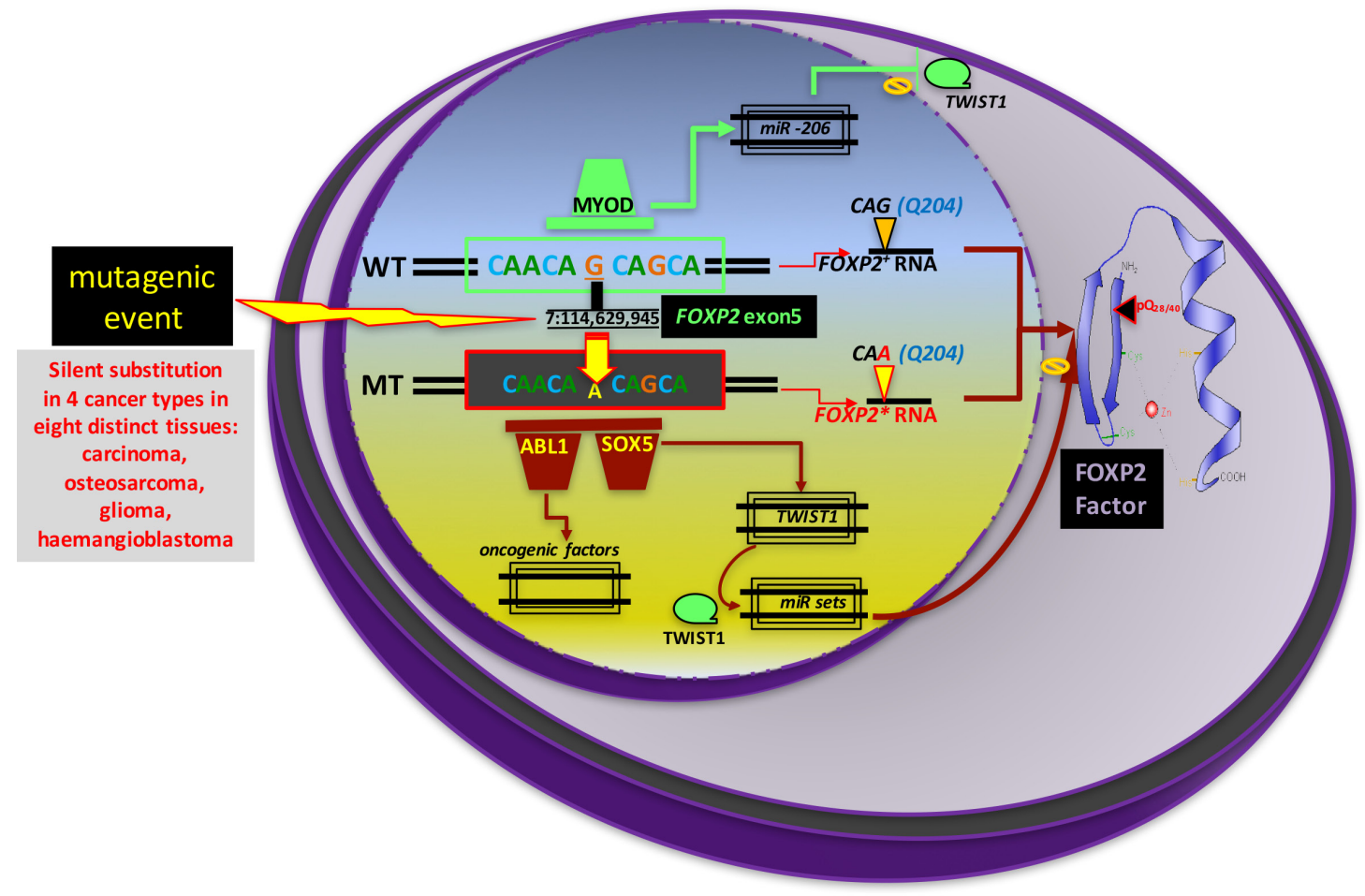

Figure 5: PROTEIN. Hypothetic scenarios of oncogenic events involving a known regulatory element embedded in the fifth exon of FOXP2 encoding the polyQ40 stretch. This proposal stems from the observation that six different cancer types, with downregulated FOXP2 expression, share an identical point mutation at 7:114,629,945 (Q204) in the fifth exon of FOXP2. This scheme explores a few of the putative functional consequences of this mutation, considering the observation that this position belongs to a validated promoter. On the one hand the mutation may hinder the fixation of an important transcription factor to this promoter. MYOD appears compatible with this site. On the other hand, this mutation may lead to the creation of a new binding site consensus for factors which normally do not bind this promoter. We represent here two compatible candidates: SOX5 (5'-TWWCAAAG-3'), and ABL1 (5'-AA/CAACAAA/C -3'). Binding of these two factors may have long-range consequences, including for instance the activation of TWIST1 by SOX5. Transcriptomic data suggest this latter scenario may prove true at least for the breast cancer $[9,178]$. 
upon interactions with a large repertoire of proteins which may have oncological consequences since some are known to behave as oncogenic drivers in cancer (see section 1.3.2). Among these, complexes including FOXP2 with nuclear receptors might be promising therapeutic candidates. For instance estrogen and androgen receptors colocalize with Foxp2 in mouse amygdala [104], and rat brain [105]. Whether this co-localization translates into real complexes remains unclear.

\subsection{Transcriptional activity upon validated FOXP2 target genes}

\subsubsection{FOXP2 target genes}

Differential expression studies across species, including human, non-human primates and animal models, have uncovered vast arrays of FOXP2 transcriptional targets, with bona fide binding sites and functional validation.

In humans two comprehensive studies on FOXP2 target genes have detected 175 and 144 targets in the developing basal ganglia (BG) and in the inferior frontal cortex, respectively (with a 24\% overlap); 192 targets in lung tissue (with a $47 \%$ and $37 \%$ overlap with BG and inferior frontal cortex, respectively) [75]; and 303 targets in human neuron-like cells (with a $14-19 \%$ overlap with BG and inferior cortex) [78]. These studies support an involvement of FOXP2 in a diversity of regulatory networks, and some of them may be time and tissuespecific.

To date, most of the studies on FOXP2 targets have been carried out in neural cells or brain tissue, focusing upon the role of FOXP2 in language. There are also some studies on lung [75] and kidney cells [80,106]. Merging all putative FOXP2 targets reported in different tissues, cellular models and mutant models in several species at different developmental stages, we collated more than 1,000 direct or indirect targets genes reported from genome-wide expression studies $[17,75,77,78,81,82]$, as well as individual gene studies [80,106,108-111]. The core analysis for the list of this set of merged targets using Ingenuity Pathway Analysis software (IPA 2017, Qiagen), provided some significant results - in particular, cancer appeared as the top disease. Among the five predominant canonical pathways we noticed the following two:

- VDR/RXR which regulates calcium/phosphorus metabolism and parathyroid hormone secretion, and is involved in immune function and tumor suppression (IPA reports).

- Wnt/b-catenin pathway which is involved in body patterning during development, cellular proliferation, differentiation and apoptosis, and is tightly associated with several cancer entities [112].

Additionally, IPA analysis reported targets of FOXP2 participating in other regulatory pathways including: inflammation, MAPK, Notch, Retinoic acid, Insulin-like growth factor, STAT3, PIK3K/Akt, CREB and TP53. Most of these results are in agreement with previous references $[63,75,78]$. FOXP2 has also been reported to interact with the Sonic Hedgehog (SHH) pathway [108].

Several studies have described FOXP2 displaying a dual functionality: mostly acting to repress expression, but also activating certain genes. The next paragraphs survey target genes reported to be repressed or activated by $F O X P 2$, and collate only candidates individually validated by means of qRT-PCR or in situ hybridization.

\subsubsection{Transcriptional repressive activity}

FOXP2 has been reported to act mainly as a repressor [75,78]. Indeed, the zinc-finger domain of FOXP2 confers transcriptional repressive properties and additionally, FOXP2 interacts with co-repressors, such as CtBP-1 [66,113]. It has been speculated that the repressive activity of FOXP2 might require integration in a multiproteic complex with members from its own family and/or from others such as CtBP-1 [114].

A list of individually validated targets directly or indirectly repressed by FOXP2 is summarized in Table 1 . In these studies, genes were selected to investigate the role of FOXP2 in language and neurodevelopment and were mostly assessed in neuronal cell models. We analyzed these genes with Ingenuity Pathway Analysis software: thirty-three of them were involved in tumorigenesis of carcinoma, with high levels of ASCL1 and MET in neuroblastoma formation [115-117], and PTCH1 in medulloblastoma initiation [118]. Moreover, these genes were also involved in the oncogenic progression of primitive neuroectodermal tumor in IPA analysis. Another gene involved in multiple cancers, CD164, has been reported to promote glioma via the tumor-suppressor PTEN [119]. These data suggest that FOXP2 may have the capacity to repress both pro-oncogenic and tumor suppressor genes. Thus, a putative FOXP2-mediated derepression of targets in oncogenic conditions may be complex, occurring only in some phases of the malignancy development, or be tissue/target specific.

\subsubsection{Transcriptional activating activity}

FOXP2 has also been reported to activate expression for some genes in several experiments. However, since these experiments were carried out upon FOXP2 overexpression in culture conditions, it cannot be ruled out that the observed activation of targets resides in the fact that other members of the FOXP2 family are competitively displaced by a surplus of FOXP2, which may impede the formation of multiproteic repressor complexes [114]. Transcriptional activation by FOXP2 may also be explained by differential affinity of FOXP2 for the DNA binding site, cofactors interacting with FOXP2 or posttranslational modifications of FOXP2 [114].

Analysis of individually validated genes upregulated by FOXP2 (Table 1) using IPA revealed cancer as the 
Table 1: Individually validated targets directly or indirectly regulated by FOXP2. Similar colour code was used for a same reference showing both activated and repressed targets by FOXP2. In bold, targets involved in oncogenesis according to IPA analysis. Such a wide variety of targets involved in cancer suggests that dysregulated FOXP2 may cause complex interaction-dependent effects during oncogenic progression.

\begin{tabular}{|c|c|c|c|c|c|}
\hline & Species & Gene & Model & Technique & References \\
\hline \multirow{12}{*}{ 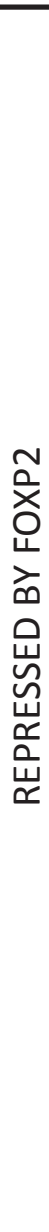 } & \multirow[t]{8}{*}{ Human } & $\begin{array}{l}\text { SLC17A3, CALCRL, LNPEP, HSPB7, } \\
\text { COX11, PM5, PSEN2, CD164, RCN2 }\end{array}$ & $\begin{array}{l}\text { Human neurons (SH-SY5Y cells) } \\
\text { transfected with FOXP2 (pcDNA3.1/ } \\
\text { FOXP2) or EMPTY vector in stable } \\
\text { culture. }\end{array}$ & $\begin{array}{l}\text { qRT-PCR } \\
\text { EMSA } \\
\text { validation }\end{array}$ & (Vernes et al., 2007) \\
\hline & & NOS1, LBR, KCNJ15, ANK1 & $\begin{array}{l}\text { SH-SY5Y cells transfected } \\
\text { with empty vector or FOXP2 isoform I. }\end{array}$ & $\begin{array}{l}\text { qRT-PCR } \\
\text { Binding } \\
\text { analysis, ChIP }\end{array}$ & (Spiteri et al., 2007) \\
\hline & & CNTNAP2 & $\begin{array}{l}\text { SH-SY5Y cultured cell expressing } \\
\text { different FOXP2 levels. }\end{array}$ & qRT-PCR & (Vernes et al., 2008) \\
\hline & & MET mRNA and protein level & $\begin{array}{l}\text { Overexpressed FOXP2 in } \\
\text { undifferentiated NHNP } \\
\text { Cells. MET expression examined } 4 \mathrm{~d} \\
\text { later. }\end{array}$ & qRT-PCR / WB & (Mukamel et al., 2011) \\
\hline & & $\begin{array}{l}\text { RORg, RXRa, TNR, DGAT1, SLIT1, BATF3, } \\
\text { AQP1, ASCL1, CNTNAP2, DLL3 }\end{array}$ & $\begin{array}{l}\text { Stable SHSY5Y cells expressing human } \\
\text { FOXP2 or the empty vec- tor (EMPTY). }\end{array}$ & qRT-PCR & (Devanna et al., 2014) \\
\hline & & $\begin{array}{l}\text { CER1, SFRP4, WISP2, SNW1, EFNB3, } \\
\text { and SLIT1 in the presence of FOXP2 } \\
\text { homodimers }\end{array}$ & $\begin{array}{l}\text { FOXP1/2/4 stably transfected into } \\
\text { Embryonic kidney cell line HEK } 293 \text { cells. }\end{array}$ & qRT-PCR & (Sin et al., 2015) \\
\hline & & SRPX2, UPAR & $\begin{array}{l}\text { Embryonic HEK293 cell line } \\
\text { transfected with FOXP2. }\end{array}$ & qRT-PCR & (Roll et al., 2010) \\
\hline & & $\begin{array}{l}\text { DISC1 promoter activity and protein } \\
\text { expression }\end{array}$ & $\begin{array}{l}\text { Embryonic kidney cell line HEK293 } \\
\text { transfected with FOXP2. }\end{array}$ & $\begin{array}{l}\text { Luciferase } \\
\text { assay / WB }\end{array}$ & (Walker et al., 2012) \\
\hline & \multirow[t]{3}{*}{ Mouse } & $\begin{array}{l}\text { Acvr2a, Efnb2, Wasf1, Foxn2, Nfat5, } \\
\text { Nptn, Nrn1 }\end{array}$ & Neuroblastoma (neuro2a) cells in vitro. & qRT-PCR & (Vernes et al., 2011) \\
\hline & & Evf1/2, Nell2, Nrn1, Cck, Lmo4 & $\begin{array}{l}\text { Different brain areas in homozygous } \\
\text { mice not expressing Foxp2 protein } \\
\text { (Foxp2-S321X) compared with WT. }\end{array}$ & $\mathrm{ISH}$ & (Vernes et al., 2011) \\
\hline & & $\begin{array}{l}\text { Ptch1 mRNA and protein level (Sonic } \\
\text { Hedgehog pathway) }\end{array}$ & P19 cells transfected & qRT-PCR / WB & (Chiu et al., 2014) \\
\hline & Rat & SRPX2 protein level & $\begin{array}{l}\text { Dissociated cortical neurons } \\
\text { electroporated with FoxP2 }\end{array}$ & WB & (Sia et al., 2013) \\
\hline \multirow{6}{*}{ 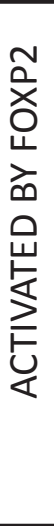 } & \multirow[t]{4}{*}{ Human } & MAPK8IP, SYK & $\begin{array}{l}\text { Human neurons (SH-SY5Y cells) } \\
\text { transfected with FOXP2 (pcDNA3.1/ } \\
\text { FOXP2) or EMPTY vector in stable } \\
\text { culture (n.s. in transient culture). }\end{array}$ & qRT-PCR & (Vernes et al., 2007) \\
\hline & & CALCRL & $\begin{array}{l}\text { SH-SYSY cells transfected } \\
\text { with empty vector or FOXP2 isoform I }\end{array}$ & qRT-PCR & (Spiteri et al., 2007) \\
\hline & & $\begin{array}{l}\text { RARb-001, RARb-002, RARb-005-201, } \\
\text { RORb, CRABP II, RGS2, SPOCK1, FGF1, } \\
\text { ID2, TJP2, NEDD9, SYK, BCL2, ETV1, }\end{array}$ & $\begin{array}{l}\text { Stable SHSY5Y cells expressing human } \\
\text { FOXP2 or the empty vec- tor (EMPTY) }\end{array}$ & qRT-PCR & (Devanna et al., 2014) \\
\hline & & $\begin{array}{l}P R I C K L E 1, N C O R 2, N E U R O D 2 \text { and PAX3 } \\
\text { in the presence of FOXP2 homodimers }\end{array}$ & $\begin{array}{l}\text { FOXP1/2/4 stably transfected into } \\
\text { Embryonic kidney cell line HEK293 cells }\end{array}$ & qRT-PCR & (Sin et al., 2015) \\
\hline & \multirow[t]{2}{*}{ Mouse } & let7-d, mir 9, mir216 & Neuroblastoma (neuro2a) cells in vitro. & qRT-PCR & (Vernes et al., 2011) \\
\hline & & PDGFRa & $\begin{array}{l}\text { CTX progenitor cells isolated from } \\
\text { the forebrain of mouse embryos } \\
\text { electroporated with US2-Foxp2. }\end{array}$ & $\mathrm{IHC}$ & (Chiu et al., 2014) \\
\hline
\end{tabular}

top listed disease. Highlighted genes involve $B C L 2$ and HES1, which are involved with proliferation of neuroblastoma cell lines, and NEUROD2 which plays a role in neurogenesis of carcinoma cell lines. Additionally, NAV2 induces neurite outgrowth and is highly expressed in neuroblastoma cells, uterine endometrial stromal sarcoma and colorectal cancer [120]. MAPK8IP plays an anti-apoptotic role [121] and showed decreased expression level in glioblastoma [122]. The protein kinase SYK has been described as tumor suppressor in breast and glial cells [123]. 


\section{Roles of FOXP2 in normal and non-oncogenic pathological conditions}

Close to sixty phenotypes have been reported across eight null mutant alleles for mouse Foxp2 (MGI:2148705). While most anatomic systems have been concerned with morphogenetic alterations, neoplasm has not been reported so far as being associated with Foxp2 dysregulation in the mouse. This observation is consistent with the reported resistance of rodents to neoplasm in general [124].

However, lack of Foxp2 entails juvenile development and leads to morphological alterations that impact CNS tissues (neocortex, cerebellum, basal ganglia), sensory organs (eye, ears), functional activities (vocalization, balance) and statuary growth with death occurring by the end of the first month due to unclear factors. The latter may include pulmonary underdevelopment, but not lack of maternal care or feeding difficulties $[20,84]$. To some extent, these defects bear some relevance to several aspects of abnormalities reported in human heterozygous patients with defective
FOXP2 expression (see section 5.2.3).

In the CNS under normal conditions, the identification of FOXP2 targets has suggested roles in neurodevelopment (neurotrophin signaling, apoptosis, differentiation, and migration) and neurotransmission (synaptic plasticity, neurite outgrowth, axonogenesis and axon guidance) $[75,78,82]$. Indeed, FOXP2 was involved in different processes such as neurogenesis [94] including production of interneurons [108], detachment of differentiating neurons from the neuroepithelium [94], neuronal differentiation [108,109,125], migration $[109,126]$, neurite outgrowth $[17,82,127]$, synaptogenesis [111] and dendritic spine morphogenesis [111,128].

Hereafter are briefly overviewed non-oncogenic defects observed in human; the cancers associated with FOXP2 dysregulation will be subsequently detailed in chapter 5 .

\subsection{FOXP2 and language}

A cardinal feature of FOXP2 hemi-deficiency in human patients pertains to language $[19,36,129]$. This

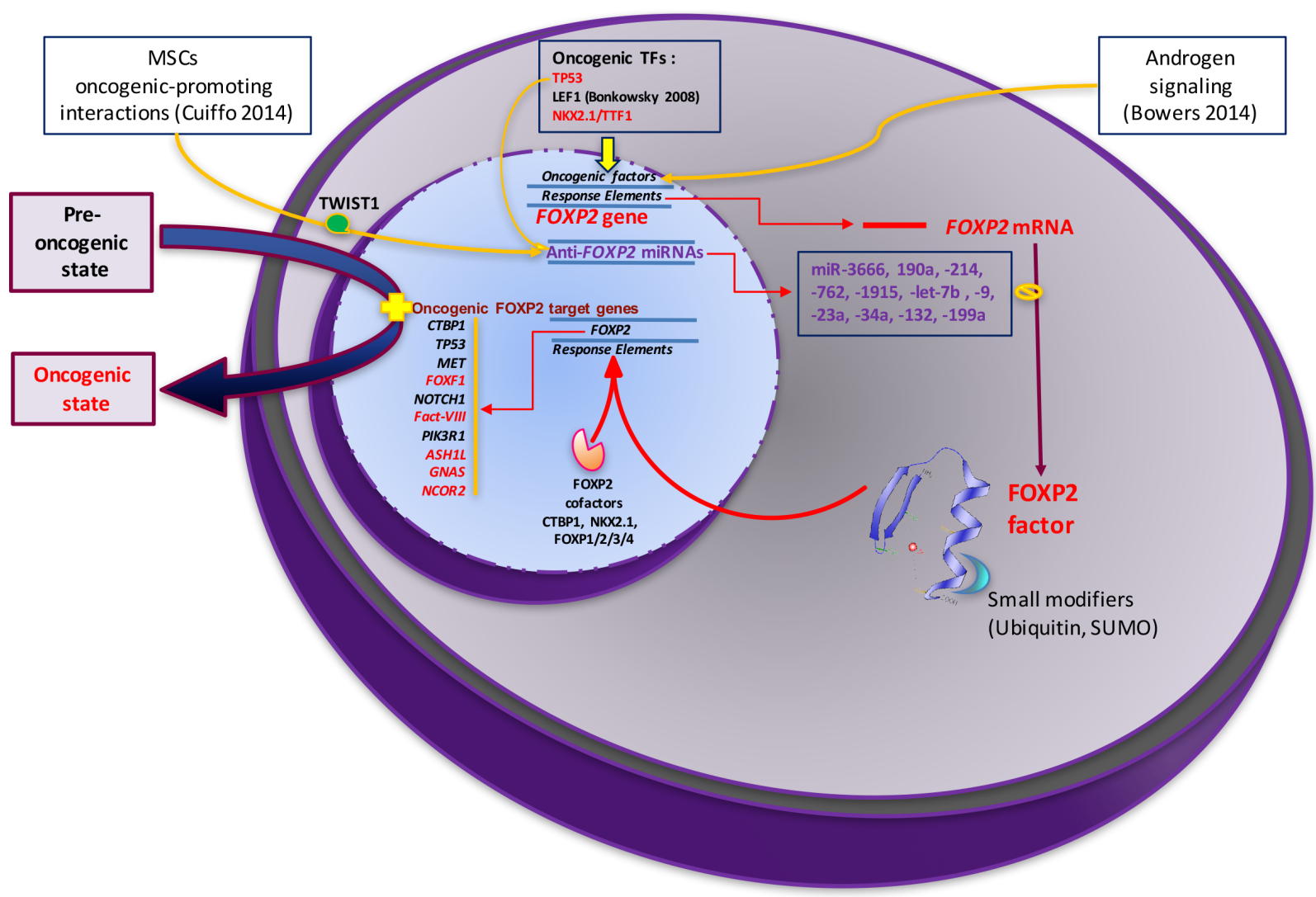

Figure 6: A putative FOXP2-dependent pro-oncogenic/tumor suppressor regulatory network. This scheme illustrates how diverse activation pathways may converge to convert a typical cell from a pre-oncogenic to an oncogenic state through abnormal FOXP2 expression and activity. Genes and factors indicated here have been observed in numerous but distinct cancer types detailed in the main text and should not be considered as collectively acting throughout all steps of the oncogenic progression. The illustration of the FOXP2 structure is from Wikipedia. 
association is detailed in the OMIM page for FOXP2 (OMIM 605317; "Speech-language disorder-1") and it was first described for the widely studied "KE" pedigree, which carries an arginine-to-histidine substitution at $\mathrm{R} 553 \mathrm{H}$ in the DNA Forkhead binding domain $[19,36]$. Both structural and functional defects impacting language were described in approximately fifteen family members. These include orofacial motor control and articulation, comprehension and expression abilities, as well as nonverbal cognitive skills, with major grammatical failure. Additional unrelated cases of people showing language deficits were also linked to heterozygous or hemizygous mutations of FOXP2 [29,130-133]. While developmental verbal dyspraxia is a fundamental disorder of this syndrome, its etiology remains debated and might be impacting multiple neural pathways $[80,134]$. Whether FOXP2 is involved throughout the construction of the neuro-musculo-skeletal apparatus bearing speech production remains debated.

\subsection{FOXP2 and autism}

We previously described the association of FOXP2 with language. Another correlation which remains to be functionally elucidated is between FOXP2 dysfunction and autism spectrum disorder (ASD). Epidemiological studies rank FOXP2 with a score of 3 on the SFARI autism scale, an intermediate "suggestive evidence" among stronger scores (Syndromic; 1: high confidence; 2: strong candidate) and weaker ones (4: minimal evidence; 5: hypothesized but untested; 6: unsupported). Importantly, its activities place FOXP2 at the center of an interactome hub regulating the expression of a cohort of other autismlinked genes. This set comprises more than 30 genes reported in different brain regions or cellular models in mouse and human, including syndromic genes (e.g. CNTNAP2, FMR1, Pax6, MEF2C), "high confidence" (e.g. TBR1), "strong candidate" genes (e.g. FOXP1, $M E T)$ and "suggestive evidence" susceptibility genes (e.g. Auts2) [75,77,78,107,109,110,135]. Additionally, large chromosome accidents comprising the FOXP2 region are functionally linked to autism (see section 5.2.3). Social communication deficits are central to ASD diagnosis, and both language dysfunction and autism may be influenced through downstream regulation by FOXP2 key target genes that ultimately impact circuit wiring [110]. One such illustrative interaction lies in the direct repression of the neurexin gene CNTNAP2 by FOXP2 upon binding to a regulatory sequence in intron 1 [81]. This neurexin has been strongly associated with autism [136], and both genes are expressed in the basal ganglia and amygdala two important territories of the social brain.

\subsection{Other neuropathogenetic processes}

Converging data indicate that $F O X P 2$ is important for modulating the plasticity of relevant neural circuits. Indeed, FOXP2 appears among the twenty-three clinically relevant genes common to ASD, bipolar disorder and schizophrenia [137]. The affected processes remain still largely unknown.

In accordance with a neurogenic role of FOXP2 in neuropathogenetic processes, one polymorphic variant of FOXP2, rs2396753, is associated with hallucinations in schizophrenia and correlated with grey matter reduction [138]. In mice, Foxp2 null mutants displayed a reduced cerebellum [20], suggesting that Foxp2 is a key regulator in the development of progenitor cell proliferation and differentiation in this territory. Moreover, reduced Foxp 2 dosage impaired motor-skill learning and synaptic plasticity in mice [139]. In fronto-temporal lobar degeneration, patients carrying FOXP2 polymorphisms affecting verbal fluency showed hypoperfusion in language-associated brain areas including the left inferior frontal gyrus, and putamen [140].

The neurogenic role of human FOXP2 was assessed in developing mouse cortical cells [141]. FOXP2 appeared to control the behaviour and fate of ventricular zone progenitors by modulating their capacity to engage into neuroglial differentiation. FOXP2 may thus act as a neurogenic switch in the embryonic brain. Lack of this switch may adversely impact neurogenesis by allowing cortical progenitors to remain in a proliferative state, a condition favoring neural oncogenesis. Whether this property subsists throughout post-natal life in brain neurogenic niches remains to be determined.

\section{Oncogenic consequences of FOXP2 dysregulation}

\subsection{Repertoire of cancers reported to involve FOXP2 dysregulation}

Attempts to assess whether FOXP2 transcript or protein levels can be of diagnostic relevance have been collated in cancer databases, along with individual cases reports. However, discrepancies still remain to be solved before a clear understanding of these conditions can be reached. Beyond inter-individual variability, these differences may suggest alternative and tissue-specific roles as tumor suppressor or as oncogene, depending on activated signaling pathways. Furthermore, whether the observed expression levels are causative or consequential to the oncogenic condition has not been systematically assessed. 
The ProteinAtlasDatabase displays FOXP2 immunodetection within twenty surveyed cancer types. We categorized these cancer conditions according to FOXP2 levels, relative to healthy tissues (Figure 4, upper panel). While six cancer types displayed unchanged levels, eight appeared moderately increased, and two strongly elevated (glioma and testicular). On the other hand of the spectrum, four conditions displayed moderately reduced FOXP2 immunosignal intensity.

In contrast, in another set of peer-reviewed studies summarized in Figure 4 (lower panel), down-regulated FOXP2 expression was found in breast cancer (RNA: [9]), hepatocellular carcinoma (protein: [8]) and gastric cancer (protein: [10]). Aberrant levels of FOXP2 factor were found in different types of prostate cancers, strong levels being linked to poor prognosis in ERG fusion-negative prostate cancers [13]. On the other hand, overexpressed FOXP2 was reported in multiple myeloma, MGUS (Monoclonal Gammopathy of Unknown Significance) and in several subtypes of lymphoma (both RNA and protein [5] or just in protein [11]) as well as in neuroblastoma (RNA and protein [12]).

While the ProteinAtlasDatabase indicates that the overall level of FOXP2 factor in twenty selected cancer conditions may vary in both directions, it thus cannot be ruled out that they reflect secondary impact of oncogenesis on FOXP2 transcription and/or translation. Genomic information should be further analyzed to complete this survey.

In the following sections ("entities") we focus upon cancer conditions associated with FOXP2 dysregulation which are supported by research articles (summarized in Figure 4, lower panel).

\subsubsection{Entity: Breast cancer cells}

Recent observations have raised a putative tumorsuppressor role for FOXP2 [9,142]. Whether this property applies to tissues different than the breast mesenchyme remains to be demonstrated. Mechanistically, FOXP2 translation appears to be actively impaired by two successive waves of dysregulated micro-RNAs: initial MSCs (mesenchymal stem cells)-induced expression of a cluster of microRNAs (miR-199a-214, miR-762) led the activation of a secondary network of microRNAs (miR1915 , let-7b, and miR-34a) which subsequently repressed the expression of FOXP2 [9].

These miRs are encoded by loci which are either intragenic (miR-1915 within CASC10 intron) or extragenic (let7b; miR-34A; miR-762 colocalized with $B C L 7 C$ intron on the reverse strand; miR-199A2 and miR-214 are both colocalized with DNM3OS and miR-199A1 on reverse strand from DNM2). Among these genes, the CASC10 is associated with cancer susceptibility, $B C L 7 C$ is a tumor suppressor and DNM2/3 are P53 activators.

The activation of the TWIST-1 transcription factor has been reported to be responsible in this cascade [9], which remains to be fully detailed. This mechanism seems to concern only breast mesenchymal stem cells, as no cancer cell population has been reported to display reduced FOXP2 transcript levels in this survey [142]. The chain of ensuing events involves the homing of these cells into the stroma of the initial breast tumor, where they mingle with quiescent cancer stem cells. This interaction potentiates the oncogenicity of cancer stem cells, which triggers a powerful growth and metastasis of the tumor.

Thus, it may be that the tumor suppressor role ascribed to FOXP2 may in fact be indirect, and rather lie within its capacity to normally prevent mesenchymal stem cells from homing into the tumor and/or subsequently activating resident cancer stem cells.

\subsubsection{Entity: Hepatocellular carcinoma (HCC)}

The Human Protein Atlas suggests a moderate or weak expression level of FOXP2 in sections from hepatocellular carcinoma biopsies. Consistently, a study proposed that reduced FOXP2 protein levels in biopsies might be associated with poor outcome [8]. In this study, established cancer cell lines were used as models to propose that such a low FOXP2 level was causative to their invasive capacity. Whether this assay reflects in vivo oncogenic processes in HCC remains to be determined as endogenous hepatic cell FOXP2 contents are already low when compared to other tissues. In line with the previously reported oncogenic mechanism (entity "breast cancer"), it might be necessary to determine whether the observed FOXP2 levels concern endogenous HCC cancer stem cells per se, or mesenchymal stem cells prone to HCC homing. Noticeably, the oncogenic course might be more complicated as it has been reported in other cancers (see further) that invasiveness was either associated with FOXP2 up-regulation as in prostate cancer [13] or, conversely, with FOXP2 down-regulation as reported for breast cancer [9]. An indirect in vitro assay in established human cell lines from hepatocellular carcinoma has shown that malignancy potential was associated with reduced levels of TALIN2 (TLN2) [54]. This gene harbors a microRNA, miR-132, which is predicted to target FOXP2 transcripts [126]. It may thus be speculated that in situ, progressive loss of TLN2, and the congruent loss of miR-132, might lead to increased levels of FOXP2 as malignancy progresses.

\subsubsection{Entity: Multiple myeloma}

FOXP2 is not detected in the normal hematopoietic lineage. Yet the discovery of its strong up-regulation in B lymphocytes from patients afflicted with hematological cancers, including multiple myeloma, has supported the proposal to add it to the arsenal of diagnostic markers, with an even better resolution than those previously available [5]. Whether this up-regulation stems from the oncogenic process or triggers it remains to be formally established in human myelomas. In particular its association with its paralog FOXP1, a strong marker of hematological 
malignancies, warrants further investigation.

\subsubsection{Entity: Diffuse large B-cell lymphoma (DLBCL)}

Browsing further blood malignancy conditions identifies FOXP2 as a putative marker for DLBCL, in line with previous observations that FOXP1 is a determinant oncogenic driver in these cancer types [11]. While both factors can be co-immunoprecipitated, whether they cooperate and synergize during transformation remains to be determined, since they might prove both to be strong target candidates as can be read in several FOXP2-related patent proposals

\subsubsection{Entity: Prostate cancers}

A large scale $(10 \mathrm{~K}+$ patients) GWAS transcriptomic survey of prostate biopsies has readily associated FOXP2 levels with cancer outcome [13]. Firstly, nuclear FOXP2 expression in epithelial cells, and not stromal cells, is reduced when compared to normal prostate epithelium. Secondly, advanced stage and severe conditions display strong epithelial staining, especially in ERG fusionnegative conditions - while ERG fusion-positive cancers lacked this association. Interestingly, FOXP2 levels were reported to significantly correlate with cancer cells proliferative activity. Thirdly, in the long-term phase of the longitudinal study, high FOXP2 levels were found to correlate with relapse frequency of ERG-negative cancers. Whether FOXP2 might be a good candidate target in these conditions remains to be determined, as its strong expression in the normal prostate epithelium might be associated with a physiologically relevant role, as for its paralogs of the FOXA family. In the meantime, this study clearly identified FOXP2 expression levels as a strongly discriminative and prognostic tool. In ERG fusion-positive prostate cancer (about $50 \%$ of cases) the androgen responsive TMPRSS2 gene fuses to the ETS family transcription factor ERG gene, increasing ERG protein expression. This may activate different pathways and hormonal interactions compared with ERG fusionnegative prostate cancer leading to differential interactions with FOXP2 and distinctive tumor progression.

With regards to oncogenesis mechanism, it should be noted that the FOXP2 transcript is a bona fide target of miR-190, a microRNA lying within an intron of, and concomitantly expressed with, the cytoskeleton-associated protein TALIN2 (TLN2) - with both genes being downregulated in advanced prostate cancer [143]. Furthermore, TLN2 hosts another microRNA, miR-132, which also targets $F O X P 2$ transcripts, and has been widely reported as being involved during prostate cancer progression [144].

An encouraging report has identified another FOXP2-targeting microRNA, miR-628, as an efficient tool to damper the aggressiveness of prostate epithelial cancer cell lines [145].

\subsubsection{Entity: Gastric cancer}

Human gastro-intestinal tract cells express FOXP2, essentially in the glandular compartment. Gastric cancer biopsies display mostly reduced FOXP2 immunoreactivity. Established Human cells lines of the gastric cancer lineage have been shown to display reduced FOXP2 transcript levels when compared to non-cancerous gastric cells [10]. Which factors may account for reduced FOXP2 expression level in gastric cancer remains to be determined. Candidates, among others, include FOXP2targeting microRNAs, whether alone or in combination. One, miR-190, has been reported to be up-regulated in both gastric cancer biopsies and in one established gastric cancer cell line [10]. As reported in other sections, miR-190 is often found associated with tumorigenic conditions. One assumption is thus that elevated miR190 might lead to reduced target FOXP2 transcripts, as observed in established cell lines [55]. However, whether this event is oncogenic to or consequential from gastric cancer development, and whether and when other FOXP2targeting miRs might be involved, remain two major issues to be solved.

\subsubsection{Entity: Glioma}

FOXP2 is involved in several aspects of central nervous system ontogenesis, including neural differentiation, axonogenesis, dendritic spine growth, synaptogenesis, neuroblast migration and synaptic plasticity (described in section 4.3). A comparative analysis of neurogenic functions exerted by FOXP2 in human and mouse has unveiled a new, human-specific activity in the developing cerebral cortex progenitor cells: in contrast to its murine orthologue, FOXP2 exerts proneurogenic activities by promoting the differentiation of human neural precursors and preventing or delaying their proliferation [141]. This property may bear oncogenic consequences since FOXP2 invalidation relieves neural progenitors from a proliferation repressive signal. This may be related to the etiological event observed in glioblastoma and neuroblastoma associated with FOXP2 dysregulation $[10,12,55]$, as a side-effect to its role in the acquisition of a stronger neurogenic contingent in the human brain.

In another study examining the pro-apoptotic effect of TP53 activation on transformed glioma cells, we noticed the downregulation of FOXP2 during cell death of these cancer cells $[146,147]$. Further studies are required to determine whether such a tumor-suppressing role, under the control of TP53, bears some relevance with FOXP2 in vivo function.

\subsubsection{Entity: Colorectal cancer}

We surveyed longitudinal reports for oncogenic conditions involving FOXP2 deficiency, either repressed or overexpressed, to assess whether it could be considered as a pro-oncogenic player. Although such reports remain 
scarce, a paper might hold relevant cues [148]: in this paper authors have examined a colorectal adenoma which progressed into a carcinoma condition, by assembling the transcriptomic signature throughout the transition. Among relevant genes, FOXP2 appeared to be the ninth-most increased among 305 up-regulated genes in precancerous tissue (adenoma), suggesting that it may belong to a group of early genes whose strong expression level was associated with a poor prognosis of tumorigenic progression. We detailed the evolution of the transcriptome throughout this process in section 5.3.2 to illustrate how FOXP2 dysregulation may associate with large sets of putative FOXP2 target genes during oncogenesis.

Collectively, this work suggests that FOXP2 in this cancer might belong to a small group of pro-oncogenes associated with "priming" the epithelium for cancer progression. Whether this observation stands true for other conditions, it remains to be elucidated by further largescale expression signatures in pre- or early malignancy conditions. This knowledge may contribute to improve our understanding of oncogenesis and provide candidate genes for diagnosis and prognosis in biopsies, along with putative therapeutic targets.

\subsubsection{Entity: Osteosarcome}

In the mouse embryo, FoxP 2 is regionally expressed in the healthy bone by proliferating chondrocytes, collar bone periostal cells but not mature osteoblasts [149]. Consistently, the human osteoblasts lacked FOXP2 expression. Functional assays on established cell lines from the bone linage suggested that at least in vitro, growth arrest resulted from strong FOXP2 upregulation through indirect induction of the cell-cycle inhibitor $p 21^{C I P / W A F 1}$, a p53 target. It is difficult at this point to connect these in vitro data with the mechanisms underlying osteosarcoma formation in human [150].

\subsubsection{Entity: Other oncogenic conditions}

This review focuses on published reports of FOXP2 involvement at various stages of oncogenic processes in human tissues. Several databases display FOXP2 expression levels in other cancer types, which we elected to mention, because no associated articles were available at the time of preparation of this review. They include testicular cancer and renal cancer (e.g. Figure 4, upper panel).

\subsection{Genome-wide and clinical oncogenomic research involving the FOXP2 locus}

Oncogenic conditions have been analyzed with regards to a genomic involvement of either the FOXP2 locus or regulatory elements controlling FOXP2 transcription:

\subsubsection{Molecular conditions preserving from oncogenesis}

Transcriptomic profiling of tumor cells has led to the discovery that cancer stem cells which display a severe malignant and highly metastatic phenotype expressed reduced FOXP2 levels with regards to normal cells. Functional validation has led to the notion that FOXP2 repression, mediated by microRNAs, was causal to this phenotype and not consequential, at least in breast cancer [9]. As discussed in paragraphs dedicated to breast cancer and colorectal entities, these observations have raised the possibility that FOXP2 might actively be part of a protective network of factors involved in preventing prooncogenic processes - at least in some tissues.

\subsubsection{Molecular conditions associated with oncogenesis}

\subsubsection{The 7:114,629,945 cancer mutation}

To determine whether variations in the genomic sequence spanning the FOXP2 locus were associated with tumorigenesis, we combined data from Ensembl and the Cosmic catalogue (release 81). We extracted phenotypic descriptions associated with 24,804 annotated variants (among $3 \mathrm{M}+$ detected), including 1,155 associated with pathogenic conditions rated "significant".

On the other hand, browsing the Cosmic catalogue returned 531 tumor biopsies displaying a confirmed somatic mutation within the FOXP2 locus. Among those, the top ten most frequent mutations appeared in at least four histological types of cancers (carcinoma, glioma, haemangioblastoma and osteosarcoma) involving eight different tissues (bone, brain, bladder, GI tract, urinary, lung, prostate, thyroid) throughout the survey, respectively up to 22, 19 and 19 times for the three most frequent variants detected at position 7:114,629,945. Interestingly, these mutations (all being $\mathrm{G}>\mathrm{A}$ substitutions) are silent, as they preserve the Q (aminoacid 204) normally encoded within the exon 5. In Figure 5 we propose a few functional consequences of this mutation.

Importantly, these substitutions neither create nor eliminate donor or acceptor consensus splice sites in the region. We note that this $\mathrm{G}(114,629,945)$ is also the first nucleotide of the FOXP2-219 isoform, a 309nt processed transcript of unknown function (ENST00000634372.1). The pathogenicity of these mutations may thus more probably lie in the chromosomal importance of this $7: 114,629,945$ nucleotide, and not with its subsequent codon translation.

To address this issue, we examined the genomic context of this position. Using Genomatix software (3.9) we identified putative binding sites which belong to an experimentally validated promoter - located at position [114,628,854..114,630,045], Genomatix Promoter ID GXP_6756854 on the plus strand. This promoter controls the transcription of FOXP2 transcript short isoforms FOXP2-225 and -219. Analyzing consensus transcription factors binding sites in this territory, involving the 
7:+114,629,945 ' $G$ ' position, yields the myoblast determining factor MYOD (CAGC) as the strongest candidate. In other words, these silent $\mathrm{G}>\mathrm{A}$ substitutions detected in numerous tumors may alter MYOD binding onto this FOXP2 promoter. At least two oncogenic scenarios might be at play.

Firstly, this mutated promoter may be unable to control the transcription of the neighboring FOXP2-225 and -219 transcripts. The first is protein-coding, while the second is processed but not translated. In this scenario, one may speculate upon a putative suppressor-like function normally preventing oncogenesis.

Alternatively, this mutated regulatory element may be unable to control longer-distance effector genes, which themselves normally bear anti-oncogenic functions. Whether MYOD exerts any role in either scenario remains to be determined. This issue might be relevant, as MYOD has been reported to exert tumor suppressor functions in numerous tissues involving FOXP2 mutation: MYOD exerts anti-proliferative actions, and is suspected to play a tumor suppressor role in breast cancer cells [151], as well as in medulloblastoma [152], and in rhabdomyosarcoma of the prostate [153]. We propose that although the MYOD gene may not be directly impacted by the oncogenic mutations in these three conditions, the MYOD factor might well be rendered functionally deficient due to its incapacity to bind its mutated target sequence in the GXP_6756854 promoter within the FOXP2 locus. Thus, this hypothetical oncogenic scenario at play may involve the lack of function of MYOD due to a silent oncogenic mutation within FOXP2. Experimental evidence is required to assess this hypothesis. This mechanism involving MYOD may not be in itself of sufficient oncogenic relevance.

Indeed, in parallel, the conversion of this MYOD binding site into one specific for another transcription factor may confer a new binding target for alternative factors. We have found that SOX5 may be a suitable candidate transcription factor accommodating for this new site. Such a conversion may allow for a newly defined transcriptional control of an alternative set of target genes involved in oncogenesis. Here we have illustrated such a possibility with TWIST1, a bona fide SOX5 target on the same chromosome as FOXP2 [154]. TWIST1 may trigger a chain of regulatory events through the activation of miRs; subsequently, this may lead to FOXP2 downregulation [9].

Another possibility relates to the capacity of the pro-oncogenic factor ABL1 to bind the same sequence. Noticeably, the ABL1 consensus is close to that of HMG-like proteins including LEF-1 and SRY (SOX5). Deciphering the subsequent chain of regulatory events triggered by the binding of ABL1 to this new site would require expensive experimental evidence given the wide array of oncogenic roles exerted by ABL1 $[155,156]$.

Altogether these proposed steps may explain how a single mutation within $F O X P 2$ may indirectly become a genetic determinant of oncogenesis without affecting FOXP2 simultaneously in six different types of cancers.

\subsubsection{Other putative FOXP2 loci of oncogenic interest}

A pair of FOXP2 binding regions in the MET locus is located at the end of the third intron (in hGRC38 coordinates 7:116,738,696-116,738,727 =caaattaggtactttgagaatcttcccaaatt), which corresponds to the new coordinates of the intronic site reported elsewhere [110]. We found the exact same sequence at $7: 116,312,355-116,312,386$, which is reported to match HGFR isoforms a/b pre-proteins (synonymous to MET), but also falls within the locus of the caveolin $C A V 2$ gene, further centromeric to MET and FOXP2 on chromosome 7. Dysregulation of this gene has been involved, together with its neighboring paralog $C A V 1$, in numerous oncogenic processes [157].

Alternatively, these mutations may slightly impart a consensus binding site (CAGGATAATGA), for the POU6F1 factor, for which oncogenic involvement has been reported [158]. Thus, while not affecting directly FOXP2 factor function, these three most frequently surveyed mutations identify the FOXP2 locus as a critical region for the prevention of oncogenesis through the action of an intragenic promoter. This may relate to other detected variations within the human lineage for the FOXP2 sequence, which involve a POU3F2 binding site having a cis-regulatory role $[44,159]$.

In contrast, missense somatic mutations are much less frequent; the first appearing in the list, with three occurrences, being R553W at position 7:114,659,632. Other studies at this position have already identified it as critical for the proper nuclear translocation of FOXP2 a widely known allele being the $\mathrm{R} 553 \mathrm{H}$ variant reported throughout the pedigree of the language deficient "KE" family (described in section 4.1). One may speculate that in skin cells, FOXP2 exerts an anti-proliferative role which is impaired by the mutation - which may be determinant for skin oncogenesis. It remains to be determined whether FOXP2 R553W can translocate into the nucleus of affected skin cells or translocation is dampened as for $\mathrm{R} 553 \mathrm{H}$ (see section 1.3.1).

The mutational profile in FOXP2 within cancers assembled in the Intogen database, displays an accumulation of mutations in the 7:114,270,000$114,270,020$ region. Scanning this territory suggests a TAAT box, among others, but no further salient functional motifs. Genomatix analysis of this territory does not identify a validated promoter region, and PROMO.com detection of consensus binding sites identifies a series of putative transcription factors. Among those, TFII, GATA and C/EBP families might appear as the most interesting ones with regards to either transcriptional initiation or oncogenesis, respectively. Further detailed analysis of this territory falls beyond the scope of this review. 
Computing cumulative occurrences of mutations across tissue types provides an interesting cue for the oncogenic relevance of FOXP2. Using INTOGEN we compared those of FOXP2 with those of the TP53 tumor suppressor gene. Most cancer types displayed a 70-90\% range of frequencies for TP53, which is consistent with its ubiquitous expression and anti-oncogenic role. In contrast, FOXP2 was associated with an $8-10 \%$ score at most in digestive and neural cancers - and averaged a few percent across the rest of the panel. This comparison, while not ruling out a protective role for $F O X P 2$, may be indicative of a more restricted role in preventing cancer initiation.

In conclusion, while our analysis highlights variants which have been detected in tumor biopsies, it remains to be formally demonstrated that these are causative oncogenic events.

\subsubsection{Large chromosomal accidents not associated with oncogenesis}

To survey the involvement of the FOXP2 locus in oncogenic conditions reported in human patients, we browsed the databases detailed below, using either "FOXP2" or "HSA7:[114,063,327 .. 114,693,777]" as query terms. To date (February 2018), in DECIPHER database thirty-two patients have been diagnosed with chromosomal accidents involving large portions of chromosome 7 in the vicinity of the FOXP2 locus: (i) Four of these hits are punctual and three within the 7:114,086,327-114,693,772bp FOXP2 locus. One is outside at $114,066,645 \mathrm{bp}$ (close upstream to the start of several transcriptional isoforms); and (ii) the seven others span a region from 0.7 to $23.7 \mathrm{Mbp}$ located either within, covering part or entirely including this locus and its immediate neighborhood. Large chromosomal accidents spanning the $114.1-114.7 \mathrm{Mb}$ of $F O X P 2$ were specifically: Hsa7: 96.1-119.8; 113.6-126.1, 114.0-114.8, 109.1$115.1,108.8-117.6$ and 112.1-119.2Mb. The phenotypic analysis of these six patients has led to sort them into morphogenetic (craniofacial, appendage) and functional deficiencies (language, speech) as well as social disorders (mainly autism-related conditions). Despite this rather large survey of the FOXP2 locus region, none of these 32 patients have been reported so far in this database to harbor oncogenic process-related issues.

As an alternative source of oncogenic data related to lesions affecting chromosome 7, we filtered the database Chr7.org [160] for the FOXP2 locus and the occurrence of neoplastic phenotypes. However, the seven reported cases to the last update (August 18, 2004) appear either spared from, or have not been diagnosed with such malignant process -their phenotype being mainly associated with language deficits.

Over the past decade efforts have been produced to reconcile oncogenic knowledge from different databases to gain insight into the malignant processes. For deeper and updated analysis of a putative oncogenic role of FOXP2, we refer the reader to the series of gene lists compiled on the Buschman laboratory website.

\subsection{Hypothesis for a FOXP2-dependent oncogenic gene regulatory network}

\subsubsection{Putative underlying FOXP2 interactome in cancer}

The molecular mechanisms through which FOXP2 exerts its transcriptional activities in an oncogenic situation remain to be elucidated at the biochemical level.

In vitro dissection of FOXP2 dimerization with its paralogs FOXP1 and FOXP4 in human cells has shown that the fate of neuronal-specific targets genes of FOXP2 depends upon the composition of the dimers: FOXP2 associated with FOXP1 does not exert the same activity upon FOXP2 target genes than when it is dimerized with FOXP4 [64]. Whether FOXP2 is coexpressed with - and which - FOXP paralogs in pre-oncogenic cell context remains to be established. Furthermore, transcriptomic analysis of cells undergoing oncogenic transformation needs to be performed to determine whether these FOXP2 target genes are expressed and involved in the oncogenic process. These gaps in our knowledge nevertheless emphasize that the study of an oncogenic role by FOXP2 requires a global understanding of its interactions with other Forkhead factors, targets, as well as other upstream regulators including microRNAs, enhancers and cofactors as CtBP1 (described in section 1.3.1 and 3.3.2), which may modify the transcriptional regulatory activity of FOXP2. A prominent example of regulatory element controlling FOXP2 transcription is MYOD: binding onto FOXP2 promoter exerts anti-proliferative actions (section 5.1). Anomalous regulatory activity of FOXP2 due to aberrant upstream regulation as well as to mutations in FOXP2 locus (or in regulatory elements) (see section 5.2.2) might be key in triggering oncogenesis.

Putative molecular involvement of FOXP2 during cancer initiation, maintenance and metastasis processes may relate to FOXP2 ability to differentially modify the expression of target genes linked to several signaling pathways (see section 2.3.1). Screening canonical molecular pathways for putative targets of FOXP2 we observe that from angiogenesis/neovasculogenesis to glucose metabolism and apoptosis, different cancerpromoting physiological processes might be impacted by FOXP2 dysregulation.

IPA analysis using only targets individually studied (collated in Table 1) suggests involvement of FOXP2 in several pathways dysregulated in oncogenic processes, including: NOTCH1, inflammatory response, Wnt/bcatenin, STAT3 and P53.

FOXP2 interactome includes genes from the canonical NOTCH signaling (e.g. PSEN2, DLL3, HES1) and $\mathrm{Wnt} / \mathrm{b}$-catenin signaling pathway (SFRP4, ACVR2A). All of these genes have been previously related to cancer 
conditions (IPA reports). Additionally, LEF1 and MET, tightly related to the $\mathrm{Wnt} / \mathrm{b}$-catenin signaling pathway [161], are strongly associated to FOXP2 (for $L E F 1$ see section 1.1.3.2) (for $M E T$ see sections 1.1.1, 1.1.2.2, 5.2.2 and 5.3.3), and both of them have been proposed as biomarkers for prognosis and targets for cancer treatment [41] [24].

Tumor development requires vascularization of the area for the supply of nutrients, primarily glucose. FOXP2 has been reported to upregulate $B C L 2$ [109], involved in the VEGF pathway (mediating vascularization), as well as in glucose metabolism and glucocorticoid receptors, in tumor suppressor P53 pathway and inflammatory response.

Additionally, FOXP2 interacts with genes modulating the inflammatory response through cytokines, chemokines and angiogenic factors. In cancer and epithelial cells exposed to carcinogens, cell survival and proliferation are regulated by such inflammatory response as well as from apoptotic pathways involving targets of FOXP2 including: Cyclin D, c-MYC [9] and BCL2 [179]. IPA analysis provided additional FOXP2 targets participating in inflammatory processes (e.g. NFAT5, $S Y K$, $P S E N 2, A C V R 2 A)$ as well as in glucose metabolism and glucocorticoid receptors (NFAT5, NCOR2). According to IPA analysis, several FOXP2 targets involved in oncogenesis were associated to different pathways, including: ERK/MAPK signaling (HSPB7); dopamine (NOS1, KCNJ15), SHH pathway (PTCH1) [108].

During tumor progression, dysregulation of pathways involved in embryonic development is commonly observed - mainly those associated with cell proliferation. SHH pathway is involved in cell differentiation, proliferation and tissue polarity, and is found to be hyperactivated in many solid tumors. Ectopic expression of $\mathrm{SHH}$ is sufficient to induce basal cell carcinoma in mice [26]. Among other possible associations with developmental genes, FOXP2 was reported to downregulate $P T C H 1 \mathrm{mRNA}$ and protein levels involved in SHH pathway [108].

Further functional links between FOXP2 and cancer through dysregulation of other signaling processes may warrant examination. Among those we speculate that estrogen/androgen pathways relate FOXP2 and oncogenesis [104,105] (see section 5.1.5. Prostate cancer). Estrogen/androgen pathways are crucial in glandular cancers including breast or prostate cancer. Among others, estrogen receptor signaling comprises several oncogenes targets of FOXP2, including CDK8 [82,159]. Noticeably, the FOXP2 binding partner and paralog FOXP1 was found upregulated and directly activated by estrogen signaling in both breast cancer cells and biopsies [162].

In summary, FOXP2 activity may be in a hub of different pathways which are important oncogenic contributors. The involvement of FOXP2 to activate one pathway or another in oncogenesis may depend upon its interaction with paralogs, cofactors and additional regulatory elements that require further investigation.

\subsubsection{Dysregulated FOXP2-dependent genes in cancers}

The putative involvement of FOXP2 in so many different oncogenic processes may result from an intrinsic property of this factor to differentially regulate, or cooperate with general oncogenesis-associated genes, including both proto-oncogenes and tumor suppressor genes. For the most widely reported of those genes, we examined the possibility that FOXP2 might control their transcription upon binding in their regulatory elements.

The $C$-MET proto-oncogene has been involved in numerous oncogenic conditions [163], including those for which we examined the contribution of FOXP2. Different regulatory networks may be intervening. In particular, $C$-MET expression is regulated by the p53 tumorsuppressor [24]. Additionally, MET transcription falls under the control of the AP1-NFAT complex [110,164]. AP1 binds the MET promoter at its 7:116,671,439 target binding site (cagaAATTtgagttattatagta). In contrast, FOXP2 prevents MET expression through competitive binding with AP1, at the same position (underlined in the above sequence) [110], and crystallisation of the ternary complex DNA/(NFAT-AP-1)/FOXP2 has shown that FOXP2 further impairs AP1 transcriptional competency $[165,166]$. Altogether, these data suggest that a dual oncogenic mechanism may be at play, whereby normal cellular homeostasis and oncogenesis protection exerted by MET in normal conditions might be lost, due to the competitive displacement of AP1 by FOXP2 from the MET promoter. This scenario relates strongly to the oncogenic mechanism reported in immune cancers involving FOXP3-mediated displacement of AP1-NFAT from an interleukin gene [167].

Transcriptional regulatory activity of FOXP2 may be modified by the co-factor CtBP1 (described in section 1.3.1 and 3.3.2) and this interaction may have important oncogenic consequences considering the tumor-promoting activity of CtBPs. CtBPs stimulate epithelial-mesenchymal transition, tumor cell migration and invasion, inhibit apoptosis and repress several tumorsuppressor genes. Conversely several tumor-suppressors target and downregulate CtBPs [67].

We also explored the interaction between FOXP2 and the genes involved in the progression of cancer. The differential expression of genes along the transition from normal to dysplastic epithelium and then to carcinoma has been assessed for colorectal cancer [148]. We exploited these data to draft a putative network of FOXP2dependent target genes modified before or during cancer progression. First we assembled a database of known FOXP2-associated genes (see section 3.3.1), and crossed it with a set of genes modified by at least two-fold, along the progression of colorectal cancer (CRC) malignancy [148]. However, since this last list [148] contains multiple 
entries for the same genes, without specifying their raw fold-change values, it is difficult to obtain a clear view of the oncogenic evolution of the transcriptome. This appears especially important as the values reported on the histograms do not reflect the position of the candidates according to their descending order of fold-change in the table. Overall, this lack of clarity makes the task to design a CRC stage-specific signature challenging. Interestingly, four candidates have been validated by immunocytochemistry on stage-specific biopsies, which may be helpful to start defining a diagnosis strategy. This has been performed in another study where the two most downregulated candidates, DEFA5 and DEFA6, have been independently assessed as good markers of colon cancer progression [168]. In the above mentioned CRC study, FOXP2 was highly upregulated upon transition to cancer stage [148]. This was simultaneous with downregulation of some of its target genes including: $H O X B 5, P L A 2 R 1$ and $N P T X 2$. HOXB5 has been reported to be over-expressed in various cancers and knocking it down inhibited metastasis $[169,170]$. In contrast $P L A 2 R 1$ is considered a repressed tumor-suppresssor in several oncogenic conditions [171]. Whether the downregulation of these three FOXP2 target genes is consequential to an increase in upregulated FOXP2 activity remains unclear. In contrast, we found a set of FOXP2-associated genes which were upregulated during successive steps of cancer progression. Based upon IPA assignment, these genes are involved in oncogenic processes and stages such as proliferation, primary cancer establishment, invasion and metastatic tumor: $C A L D 1$, CYR61, EPHA2, PLAUR, THBS1, ZFP36 among others.

Additionally, using Genecard we inspected the promoters of widely reported tumor suppressor genes for the presence of FOXP2 consensus binding sites. We found them in a wide number of tumor suppressor genes including: TP53, APC, RB1, VHL, BRCA1, BRCA2. These putative FOXP2-dependent genes may be involved in preventing oncogenesis in different tissues. One may speculate that an oncogenic scenario involves the loss of FOXP2-dependent regulation of these oncogenic guardians, which may subsequently favor oncogenesis.

\subsubsection{Chemotherapy and drug resistance}

We explored the possibility that FOXP2 might also exert a role during a later phase of the oncogenic process, in the active elimination of therapeutic agents by cancer cells. This activity is a hallmark of drug-resistance aggressive cancers such as glioblastoma. It is mediated by transporters which expel the chemical compounds, and belong to the $\mathrm{ABC}$ family of transmembrane shuttling proteins [172]. ABCA6 and ABCG2 showed aberrant expression in different types of cancers [173,174] and are direct FOXP2 target genes [78,82]. One oncogenic promoting scenario would be the down-regulation of FOXP2 during the initial phases of tumorigenesis, paving the way for the de-repression of the transporters. Other genes related to FOXP2 that may be involved in cancer drug resistance by drug efflux according to IPA analysis of FOXP2 targets include: FOXO1, RRAS, MAPK3, PIK3R1, $M R A S$ and $P I K 3 C B$.

\section{CONCLUSIVE REMARKS: A DUAL ROLE FOR FOXP2?}

Throughout this review we have focused upon published reports involving FOXP2 dysregulation during cancer progression. We have taken care to separate this process according to tissue type. FOXP2 is not as ubiquitous as the tumor-suppressor TP53, but its involvement in such a wide diversity of tissues may underlie a more general property of this gene. Indeed, phenotypes associated with loss- or lack-of function of FOXP2 fall into two large functional categories.

The first includes neurodevelopmental defects leading mostly to dysfunctional speech associated structures, including both intellectual and motor skills required for speech production. The second category covers oncogenic defects resulting from dysregulated FOXP2 levels. It may stem from its capacity to control cell cycle progression, cellular adhesive properties, and cancer cell aggressiveness. Overall, the central nervous system displays involvement of FOXP2 in both of these categories. In developing cerebral and cerebellar cortices, the proliferating progenitors are kept under tight neurogenic control by post-mitotic cells. Failure of this proliferation checkpoint may lay adverse conditions conducive to neuro- and gliogenic cancer progression. Simultaneously, dysregulated neurogenesis may impair proper neuro-differentiation as well as timely and functional establishment of neuronal circuits, including those recruited for language and speech production.

Aberrant expression of FOXP2 was detected in diverse cancer types, including up- or down-regulated FOXP2 levels depending on the type of cancer, with few discrepancies between peer-reviewed studies and ProteinAtlasDatabase (Figure 4). This suggests that aberrant FOXP2 levels may play either a pro-oncogenic or a deficient tumor-suppressor role that may be tissuespecific and vary along the progression of cancer. Such a dual role of FOXP2 might be ascribed to differential activation of numerous gene targets of FOXP2 and regulatory networks that could affect oncogenic phenotypes independently. Differential target gene/ pathway activation may be a consequence of a complex control of FOXP2 regulation (including micro-RNAs, different combinations of FOXP1/2/4 dimerization, isoforms, alternative splicing, post-translational SUMOylation, interaction with other transcription factors, promoters and other regulatory elements detailed in sections 1 and 5.3) combined with intrinsic binding properties of FOXP2 to different DNA sequences [65].

In the present review, we have described several 
mechanisms relating FOXP2 to carcinogenesis. They enlighten that further research on the regulation of FOXP2 and the differential downstream regulatory activity of FOXP2 may be crucial to address both roles of FOXP2 in oncogenesis, as well as in language and other neurological deficits (see section 4). This complex regulation is also supported by the fact that FOXP2 structure is among the most evolutionarily conserved (only 2 amino-acids differ between human and chimpanzee) $[17,175]$. Thus, even if conservation also applies for some regulatory elements of FOXP2 that are common in both zebrafish and humans (see section 1.1.3.2), essential differences in control mechanisms and regulatory elements of FOXP2 might explain differential downstream activity and functions of FOXP2 in "speaking" versus "non-speaking" species, as well as in oncogenic $v s$. normal conditions.

It is worth noticing that most of the studies addressing FOXP2 in cancer entities reported aberrant levels of expression without providing a causative oncogenic event. To our knowledge, only one study mechanistically linked FOXP2 with the acquisition of metastatic traits, evidencing association of down-regulated FOXP2 with malignant breast cancer [9]. Parallel comprehensive studies in other cancer types will help to better understand the roles of FOXP2 in oncogenesis.

\section{ACKNOWLEDGMENTS}

Authors wish to thank the DECIPHER Consortium, and Dr Javad Nazarian for critical reading of the manuscript.

\section{CONFLICTS OF INTEREST}

The authors declare no conflicts of interest.

\section{FUNDING}

The authors thank Dr. Joshua Corbin for support.

\section{REFERENCES}

1. Abate-Shen C. Deregulated homeobox gene expression in cancer: cause or consequence? Nat Rev Cancer. 2002; 2: 777-85.

2. Gitton Y, Levi G. DLX2 (Distal-less Homeobox 2). Atlas Genet Cytogenet Oncol Haematol. 2014; 18: 805-9.

3. Gitton Y, Levi G. DLX5 (Distal-less Homeobox 5). Atlas Genet Cytogenet Oncol Haematol. 2014; 18: 810-6.

4. Gitton Y, Levi G. DLX6 (Distal-less Homeobox 6). Atlas Genet Cytogenet Oncol Haematol. 2014; 18: 817-23.

5. Campbell AJ, Lyne L, Brown PJ, Launchbury RJ, Bignone P, Chi J, Roncador G, Lawrie CH, Gatter KC, Kusec $\mathrm{R}$, Banham AH. Aberrant expression of the neuronal transcription factor FOXP2 in neoplastic plasma cells. Br
J Haematol. 2010; 149: 221-30.

6. Katoh M, Igarashi M, Fukuda H, Nakagama H, Katoh M. Cancer genetics and genomics of human FOX family genes. Cancer Lett. 2013; 328: 198-206.

7. Myatt SS, Lam EW-F. The emerging roles of forkhead box (Fox) proteins in cancer. Nat Rev Cancer. 2007; 7: 847-59.

8. Yan X, Zhou H, Zhang T, Xu P, Zhang S, Huang W, Yang L, Gu X, Ni R, Zhang T. Downregulation of FOXP2 promoter human hepatocellular carcinoma cell invasion. Tumor Biol. 2015; 36: 9611-9.

9. Cuiffo BG, Campagne A, Bell GW, Lembo A, Orso F, Lien EC, Bhasin MK, Raimo M, Hanson SE, Marusyk A, El-Ashry D, Hematti P, Polyak K, et al. MSC-regulated microRNAs converge on the transcription factor FOXP2 and promote breast cancer metastasis. Cell Stem Cell. 2014; 15: 762-74.

10. Jia W-Z, Yu T, An Q, Yang H, Zhang Z, Liu X, Xiao G. MicroRNA-190 regulates FOXP2 genes in human gastric cancer. OncoTargets Ther. 2016; 9: 3643-51.

11. Wong KK, Gascoyne DM, Soilleux EJ, Lyne L, Spearman H, Roncador G, Pedersen LM, Møller MB, Green TM, Banham AH. FOXP2-positive diffuse large B-cell lymphomas exhibit a poor response to R-CHOP therapy and distinct biological signatures. Oncotarget. 2016; 7: 5294056.

12. Khan FH, Pandian V, Ramraj S, Natarajan M, Aravindan S, Herman TS, Aravindan N. Acquired genetic alterations in tumor cells dictate the development of high-risk neuroblastoma and clinical outcomes. BMC Cancer. 2015; 15: 514 .

13. Stumm L, Burkhardt L, Steurer S, Simon R, Adam M, Becker A, Sauter G, Minner S, Schlomm T, Sirma H, Michl U. Strong expression of the neuronal transcription factor FOXP2 is linked to an increased risk of early PSA recurrence in ERG fusion-negative cancers. J Clin Pathol. 2013; 66: 563-8.

14. Estruch SB, Graham SA, Quevedo M, Vino A, Dekkers DHW, Deriziotis P, Sollis E, Demmers J, Poot RA, Fisher SE. Proteomic analysis of FOXP proteins reveals interactions between cortical transcription factors associated with neurodevelopmental disorders. Hum Mol Genet. 2018.

15. Ferland RJ, Cherry TJ, Preware PO, Morrisey EE, Walsh CA. Characterization of Foxp2 and Foxp1 mRNA and protein in the developing and mature brain. J Comp Neurol. 2003; 460: 266-79.

16. Teramitsu I, Kudo LC, London SE, Geschwind DH, White SA. Parallel FoxP1 and FoxP2 Expression in Songbird and Human Brain Predicts Functional Interaction. J Neurosci. 2004; 24: 3152-63.

17. Enard W, Gehre S, Hammerschmidt K, Hölter SM, Blass T, Somel M, Brückner MK, Schreiweis C, Winter C, Sohr R, Becker L, Wiebe V, Nickel B, et al. A humanized version of Foxp2 affects cortico-basal ganglia circuits in mice. Cell. 2009; 137: 961-71. 
18. Staes N, Sherwood CC, Wright K, Manuel M, Guevara EE, Marques-Bonet T, Krützen M, Massiah M, Hopkins WD, Ely JJ, Bradley BJ. FOXP2 variation in great ape populations offers insight into the evolution of communication skills. Sci Rep. 2017; 7: 16866.

19. Lai CS, Fisher SE, Hurst JA, Vargha-Khadem F, Monaco AP. A forkhead-domain gene is mutated in a severe speech and language disorder. Nature. 2001; 413: 519-23.

20. Shu W, Cho JY, Jiang Y, Zhang M, Weisz D, Elder GA, Schmeidler J, De Gasperi R, Sosa MAG, Rabidou D, Santucci AC, Perl D, Morrisey E, et al. Altered ultrasonic vocalization in mice with a disruption in the Foxp2 gene. Proc Natl Acad Sci U S A. 2005; 102: 9643-8.

21. Bernheim A. Cytogenomics of cancers: from chromosome to sequence. Mol Oncol. 2010; 4: 309-22.

22. Paratala BS, Dolfi SC, Khiabanian H, Rodriguez-Rodriguez L, Ganesan S, Hirshfield KM. Emerging Role of Genomic Rearrangements in Breast Cancer: Applying Knowledge from Other Cancers. Biomark Cancer. 2016; 8: 1-14.

23. Ma K, Qiu L, Mrasek K, Zhang J, Liehr T, Quintana LG, Li Z. Common Fragile Sites: Genomic Hotspots of DNA Damage and Carcinogenesis. Int J Mol Sci. 2012; 13: 11974-99.

24. Zhang J, Babic A. Regulation of the MET oncogene: molecular mechanisms. Carcinogenesis. 2016; 37: 345-55.

25. Ma H, Weng D, Chen Y, Huang W, Pan K, Wang H, Sun J, Wang Q, Zhou Z, Wang H, Xia J. Extensive analysis of D7S486 in primary gastric cancer supports TESTIN as a candidate tumor suppressor gene. Mol Cancer. 2010; 9: 190.

26. Rimkus TK, Carpenter RL, Qasem S, Chan M, Lo H-W. Targeting the Sonic Hedgehog Signaling Pathway: Review of Smoothened and GLI Inhibitors. Cancers [Internet]. 2016 [cited 2017 Jun 17]; 8.

27. Fiskus W, Mitsiades N. B-Raf Inhibition in the Clinic: Present and Future. Annu Rev Med. 2016; 67: 29-43.

28. Yamaguchi H, Hung M-C. Regulation and Role of EZH2 in Cancer. Cancer Res Treat Off J Korean Cancer Assoc. 2014; 46: 209-22.

29. Feuk L, Kalervo A, Lipsanen-Nyman M, Skaug J, Nakabayashi K, Finucane B, Hartung D, Innes M, Kerem B, Nowaczyk MJ, Rivlin J, Roberts W, Senman L, et al. Absence of a paternally inherited FOXP2 gene in developmental verbal dyspraxia. Am J Hum Genet. 2006; 79: 965-72.

30. Thomas AC, Frost JM, Ishida M, Vargha-Khadem F, Moore GE, Stanier P. The speech gene FOXP2 is not imprinted. J Med Genet. 2012; 49: 669-70.

31. Deng G, Yang J, Zhang Q, Xiao Z-X, Cai H. MethCNA: a database for integrating genomic and epigenomic data in human cancer. BMC Genomics. 2018; 19: 138.

32. Takaki H, Kikuta R, Shibata H, Ninomiya H, Tashiro N, Fukumaki Y. Positive associations of polymorphisms in the metabotropic glutamate receptor type 8 gene (GRM8) with schizophrenia. Am J Med Genet Part B Neuropsychiatr
Genet Off Publ Int Soc Psychiatr Genet. 2004; 128B: 6-14.

33. Adegbola AA, Cox GF, Bradshaw EM, Hafler DA, Gimelbrant A, Chess A. Monoallelic expression of the human FOXP2 speech gene. Proc Natl Acad Sci U S A. 2015; 112: 6848-54.

34. Estruch SB, Graham SA, Chinnappa SM, Deriziotis P, Fisher SE. Functional characterization of rare FOXP2 variants in neurodevelopmental disorder. J Neurodev Disord. 2016; 8: 44.

35. Coolen MW, Stirzaker C, Song JZ, Statham AL, Kassir Z, Moreno CS, Young AN, Varma V, Speed TP, Cowley M, Lacaze P, Kaplan W, Robinson MD, et al. Consolidation of the cancer genome into domains of repressive chromatin by long range epigenetic silencing (LRES) reduces transcriptional plasticity. Nat Cell Biol. 2010; 12: 235-46.

36. Fisher SE, Scharff C. FOXP2 as a molecular window into speech and language. Trends Genet TIG. 2009; 25: 166-77.

37. Torres-Ruiz R, Benitez-Burraco A, Martinez-Lage M, Rodriguez-Perales S, Garcia-Bellido P. Functional genetic characterization by CRISPR-Cas9 of two enhancers of FOXP2 in a child with speech and language impairment. bioRxiv. 2016.

38. Schroeder DI, Myers RM. Multiple transcription start sites for FOXP2 with varying cellular specificities. Gene. 2008; 413: 42-8.

39. Takahashi H, Takahashi K, Liu F-C. FOXP genes, neural development, speech and language disorders. Adv Exp Med Biol. 2009; 665: 117-29.

40. Bonkowsky JL, Wang X, Fujimoto E, Lee JE, Chien C-B, Dorsky RI. Domain-specific regulation of foxP2 CNS expression by lef1. BMC Dev Biol. 2008; 8: 103.

41. Santiago L, Daniels G, Wang D, Deng F-M, Lee P. Wnt signaling pathway protein LEF1 in cancer, as a biomarker for prognosis and a target for treatment. Am J Cancer Res. 2017; 7: 1389-406.

42. Raab JR, Kamakaka RT. Insulators and promoters: closer than we think. Nat Rev Genet. 2010; 11: 439-46.

43. Bao L, Zhou M, Cui Y. CTCFBSDB: a CTCF-binding site database for characterization of vertebrate genomic insulators. Nucleic Acids Res. 2008; 36: D83-87.

44. Maricic T, Günther V, Georgiev O, Gehre S, Curlin M, Schreiweis C, Naumann R, Burbano HA, Meyer M, Lalueza-Fox C, de la Rasilla M, Rosas A, Gajovic S, et al. A recent evolutionary change affects a regulatory element in the human FOXP2 gene. Mol Biol Evol. 2013; 30: 844-52.

45. Hearing VJ, Leong SPL. From Melanocytes to Melanoma: The Progression to Malignancy. Springer Science \& Business Media; 2007. 673 p.

46. Lellahi SM. POU3f2 in human gliomas - Expression pattern and functional role. 2014 [cited 2017 Oct 1]. Available from https://bora.uib.no/handle/1956/8167

47. Momoi T, Fujita E. Heritable Neurodevelopmental Disorders and Endoplasmic Reticulum Stress. Protein Misfolding Disorders: A Trip into the ER. Claudio Hetz. 
2009. p. 88-93.

48. Tanabe Y, Fujita E, Momoi T. FOXP2 promotes the nuclear translocation of POT1, but FOXP2(R553H), mutation related to speech-language disorder, partially prevents it. Biochem Biophys Res Commun. 2011; 410: 593-6.

49. Tanabe Y, Fujiwara Y, Matsuzaki A, Fujita E, Kasahara T, Yuasa S, Momoi T. Temporal expression and mitochondrial localization of a Foxp2 isoform lacking the forkhead domain in developing Purkinje cells. J Neurochem. 2012; 122: $72-80$.

50. Mostafa SM, Murad W, Mohammad E, Islam A. Intronic Mirna Mir-3666 Modulates its Host Gene FOXP2 Functions in Neurodevelopment and May Contribute to Pathogenesis of Neurological Disorders Schizophrenia and Autism. J Appl Biotechnol Bioeng. 2017; 2: 1-17.

51. Shi H, Ji Y, Zhang D, Liu Y, Fang P. MicroRNA-3666induced suppression of SIRT7 inhibits the growth of nonsmall cell lung cancer cells. Oncol Rep. 2016; 36: 3051-7.

52. Wang G, Cai C, Chen L. MicroRNA-3666 Regulates Thyroid Carcinoma Cell Proliferation via MET. Cell Physiol Biochem. 2016; 38: 1030-9.

53. Li L, Han L-Y, Yu M, Zhou Q, Xu J-C, Li P. Pituitary tumor-transforming gene 1 enhances metastases of cervical cancer cells through miR-3666-regulated ZEB1. Tumor Biol. 2016; 37: 15567-73.

54. Fang K, Dai W, Ren Y-H, Xu Y-C, Zhang S, Qian Y-B. Both Talin-1 and Talin-2 correlate with malignancy potential of the human hepatocellular carcinoma MHCC-97 L cell. BMC Cancer [Internet]. 2016 [cited 2017 Jun 17]; 16.

55. Almog N, Briggs C, Beheshti A, Ma L, Wilkie KP, Rietman E, Hlatky L. Transcriptional changes induced by the tumor dormancy-associated microRNA-190. Transcription. 2013; 4: 177-91.

56. Beezhold K, Liu J, Kan H, Meighan T, Castranova V, Shi X, Chen F. miR-190-mediated downregulation of PHLPP contributes to arsenic-induced Akt activation and carcinogenesis. Toxicol Sci Off J Soc Toxicol. 2011; 123: 411-20.

57. Ichimi T, Enokida H, Okuno Y, Kunimoto R, Chiyomaru T, Kawamoto K, Kawahara K, Toki K, Kawakami K, Nishiyama K, Tsujimoto G, Nakagawa M, Seki N. Identification of novel microRNA targets based on microRNA signatures in bladder cancer. Int J Cancer. 2009; 125: 345-52.

58. Lowery AJ, Miller N, Devaney A, McNeill RE, Davoren PA, Lemetre C, Benes V, Schmidt S, Blake J, Ball G, Kerin MJ. MicroRNA signatures predict oestrogen receptor, progesterone receptor and HER2/neureceptor status in breast cancer. Breast Cancer Res. 2009; 11: R27.

59. Navon R, Wang H, Steinfeld I, Tsalenko A, Ben-Dor A, Yakhini Z. Novel Rank-Based Statistical Methods Reveal MicroRNAs with Differential Expression in Multiple Cancer Types. PLOS ONE. 2009; 4: e8003.

60. Ng EKO, Chong WWS, Jin H, Lam EKY, Shin VY, Yu J,
Poon TCW, Ng SSM, Sung JJY. Differential expression of microRNAs in plasma of patients with colorectal cancer: a potential marker for colorectal cancer screening. Gut. 2009; 58: 1375-81.

61. Ura S, Honda M, Yamashita T, Ueda T, Takatori H, Nishino R, Sunakozaka H, Sakai Y, Horimoto K, Kaneko S. Differential microRNA expression between hepatitis B and hepatitis $\mathrm{C}$ leading disease progression to hepatocellular carcinoma. Hepatol Baltim Md. 2009; 49: 1098-112.

62. Stroud JC, Wu Y, Bates DL, Han A, Nowick K, Paabo $\mathrm{S}$, Tong H, Chen L. Structure of the forkhead domain of FOXP2 bound to DNA. Struct Lond Engl 1993. 2006; 14: 159-66.

63. Lam EW-F, Brosens JJ, Gomes AR, Koo C-Y. Forkhead box proteins: tuning forks for transcriptional harmony. Nat Rev Cancer. 2013; 13: 482-95.

64. Sin C, Li H, Crawford DA. Transcriptional regulation by FOXP1, FOXP2, and FOXP4 dimerization. J Mol Neurosci MN. 2015; 55: 437-48.

65. Webb H, Steeb O, Blane A, Rotherham L, Aron S, Machanick P, Dirr H, Fanucchi S. The FOXP2 forkhead domain binds to a variety of DNA sequences with different rates and affinities. J Biochem (Tokyo). 2017.

66. Li S, Weidenfeld J, Morrisey EE. Transcriptional and DNA binding activity of the Foxp1/2/4 family is modulated by heterotypic and homotypic protein interactions. Mol Cell Biol. 2004; 24: 809-22.

67. Chinnadurai G. The transcriptional corepressor CtBP: a foe of multiple tumor suppressors. Cancer Res. 2009; 69: 7314.

68. Deng Y, Deng H, Liu J, Han G, Malkoski S, Liu B, Zhao R, Wang X-J, Zhang Q. Transcriptional Down-Regulation of Brca1 and E-cadherin by CtBP1 in Breast Cancer. Mol Carcinog. 2012; 51: 500-7.

69. Lalmansingh AS, Karmakar S, Jin Y, Nagaich AK. Multiple modes of chromatin remodeling by Forkhead box proteins. Biochim Biophys Acta. 2012; 1819: 707-15.

70. Mizutani A, Matsuzaki A, Momoi MY, Fujita E, Tanabe Y, Momoi T. Intracellular distribution of a speech/language disorder associated FOXP2 mutant. Biochem Biophys Res Commun. 2007; 353: 869-74.

71. Estruch SB, Graham SA, Deriziotis P, Fisher SE. The language-related transcription factor FOXP2 is posttranslationally modified with small ubiquitin-like modifiers. Sci Rep. 2016; 6: 20911.

72. Meredith LJ, Wang C-M, Nascimento L, Liu R, Wang L, Yang W-H. The Key Regulator for Language and Speech Development, FOXP2, is a Novel Substrate for SUMOylation. J Cell Biochem. 2016; 117: 426-38.

73. Post-translational modification of FOXP2, a transcriptional factor, involved in developmental verbal dyspraxia: O-022 [Internet]. [cited 2017 May 29]. Available 2017 May 29, from http://insights.ovid.com/congenital-anomalies/ conan/2007/12/000/post-translational-modification- 
foxp2/52/00029644

74. Takeuchi R, Ohtsuka K, Ochi N, Inaguma Y. Posttranslational modification of Foxp2, a transcriptional factor, involved in developmental verbal dyspraxia. Congenit Anom. 2007; 47: A16.

75. Spiteri E, Konopka G, Coppola G, Bomar J, Oldham M, Ou J, Vernes SC, Fisher SE, Ren B, Geschwind DH. Identification of the transcriptional targets of FOXP2, a gene linked to speech and language, in developing human brain. Am J Hum Genet. 2007; 81: 1144-57.

76. Bonkowsky JL, Chien C-B. Molecular cloning and developmental expression of foxP2 in zebrafish. Dev Dyn Off Publ Am Assoc Anat. 2005; 234: 740-6.

77. Konopka G, Bomar JM, Winden K, Coppola G, Jonsson ZO, Gao F, Peng S, Preuss TM, Wohlschlegel JA, Geschwind DH. Human-specific transcriptional regulation of CNS development genes by FOXP2. Nature. 2009; 462: 213-7.

78. Vernes SC, Spiteri E, Nicod J, Groszer M, Taylor JM, Davies KE, Geschwind DH, Fisher SE. High-throughput analysis of promoter occupancy reveals direct neural targets of FOXP2, a gene mutated in speech and language disorders. Am J Hum Genet. 2007; 81: 1232-50.

79. Nelson CS, Fuller CK, Fordyce PM, Greninger AL, Li H, DeRisi JL. Microfluidic affinity and ChIP-seq analyses converge on a conserved FOXP2-binding motif in chimp and human, which enables the detection of evolutionarily novel targets. Nucleic Acids Res. 2013; 41: 5991-6004.

80. Roll P, Vernes SC, Bruneau N, Cillario J, Ponsole-Lenfant M, Massacrier A, Rudolf G, Khalife M, Hirsch E, Fisher SE, Szepetowski P. Molecular networks implicated in speech-related disorders: FOXP2 regulates the SRPX2/ uPAR complex. Hum Mol Genet. 2010; 19: 4848-60.

81. Vernes SC, Newbury DF, Abrahams BS, Winchester L, Nicod J, Groszer M, Alarcón M, Oliver PL, Davies KE, Geschwind DH, Monaco AP, Fisher SE. A functional genetic link between distinct developmental language disorders. N Engl J Med. 2008; 359: 2337-45.

82. Vernes SC, Oliver PL, Spiteri E, Lockstone HE, Puliyadi R, Taylor JM, Ho J, Mombereau C, Brewer A, Lowy E, Nicod J, Groszer M, Baban D, et al. Foxp2 regulates gene networks implicated in neurite outgrowth in the developing brain. PLoS Genet. 2011; 7: e1002145.

83. Bacon C, Rappold GA. The distinct and overlapping phenotypic spectra of FOXP1 and FOXP2 in cognitive disorders. Hum Genet. 2012; 131: 1687-98.

84. Shu W, Lu MM, Zhang Y, Tucker PW, Zhou D, Morrisey EE. Foxp2 and Foxp1 cooperatively regulate lung and esophagus development. Dev Camb Engl. 2007; 134: 19912000.

85. Sollis E, Graham SA, Vino A, Froehlich H, Vreeburg M, Dimitropoulou D, Gilissen C, Pfundt R, Rappold GA, Brunner HG, Deriziotis P, Fisher SE. Identification and functional characterization of de novo FOXP1 variants provides novel insights into the etiology of neurodevelopmental disorder. Hum Mol Genet. 2016; 25 : 546-57.

86. Banham AH, Boddy J, Launchbury R, Han C, Turley H, Malone PR, Harris AL, Fox SB. Expression of the forkhead transcription factor FOXP1 is associated both with hypoxia inducible factors (HIFs) and the androgen receptor in prostate cancer but is not directly regulated by androgens or hypoxia. The Prostate. 2007; 67: 1091-8.

87. Bates GJ, Fox SB, Han C, Launchbury R, Leek RD, Harris AL, Banham AH. Expression of the forkhead transcription factor FOXP1 is associated with that of estrogen receptorbeta in primary invasive breast carcinomas. Breast Cancer Res Treat. 2008; 111: 453-9.

88. Fox SB, Brown P, Han C, Ashe S, Leek RD, Harris AL, Banham AH. Expression of the forkhead transcription factor FOXP1 is associated with estrogen receptor alpha and improved survival in primary human breast carcinomas. Clin Cancer Res Off J Am Assoc Cancer Res. 2004; 10: 3521-7.

89. Giatromanolaki A, Koukourakis MI, Sivridis E, Gatter $\mathrm{KC}$, Harris AL, Banham AH. Loss of expression and nuclear/cytoplasmic localization of the FOXP1 forkhead transcription factor are common events in early endometrial cancer: relationship with estrogen receptors and HIF-1alpha expression. Mod Pathol Off J U S Can Acad Pathol Inc. 2006; 19: 9-16.

90. Li C, Tucker PW. DNA-binding properties and secondary structural model of the hepatocyte nuclear factor 3/fork head domain. Proc Natl Acad Sci U S A. 1993; 90: 11583-7.

91. Takayama K, Horie-Inoue K, Ikeda K, Urano T, Murakami $\mathrm{K}$, Hayashizaki Y, Ouchi Y, Inoue S. FOXP1 is an androgen-responsive transcription factor that negatively regulates androgen receptor signaling in prostate cancer cells. Biochem Biophys Res Commun. 2008; 374: 388-93.

92. Toma MI, Weber T, Meinhardt M, Zastrow S, Grimm M-O, Füssel S, Wirth MP, Baretton GB. Expression of the Forkhead transcription factor FOXP1 is associated with tumor grade and Ki67 expression in clear cell renal cell carcinoma. Cancer Invest. 2011; 29: 123-9.

93. Zhang Y, Zhang S, Wang X, Liu J, Yang L, He S, Chen L, Huang J. Prognostic significance of FOXP1 as an oncogene in hepatocellular carcinoma. J Clin Pathol. 2012; 65: 52833.

94. Rousso DL, Pearson CA, Gaber ZB, Miquelajauregui A, Li S, Portera-Cailliau C, Morrisey EE, Novitch BG. Foxp-mediated suppression of $\mathrm{N}$-cadherin regulates neuroepithelial character and progenitor maintenance in the CNS. Neuron. 2012; 74: 314-30.

95. Howarth K, Blood K, Ng B, Beavis J, Chua Y, Cooke S, Raby S, Ichimura K, Collins V, Carter N, Edwards P. Array painting reveals a high frequency of balanced translocations in breast cancer cell lines that break in cancer-relevant genes. Oncogene. 2008; 27: 3345-59. 
96. Teufel A, Wong EA, Mukhopadhyay M, Malik N, Westphal H. FoxP4, a novel forkhead transcription factor. Biochim Biophys Acta. 2003; 1627: 147-52.

97. Zhang Y, Chen K, Sloan SA, Bennett ML, Scholze AR, O'Keeffe S, Phatnani HP, Guarnieri P, Caneda C, Ruderisch N, Deng S, Liddelow SA, Zhang C, et al. An RNA-Sequencing Transcriptome and Splicing Database of Glia, Neurons, and Vascular Cells of the Cerebral Cortex. J Neurosci. 2014; 34: 11929-47.

98. Carney RSE, Mangin J-M, Hayes L, Mansfield K, Sousa VH, Fishell G, Machold RP, Ahn S, Gallo V, Corbin JG. Sonic hedgehog expressing and responding cells generate neuronal diversity in the medial amygdala. Neural Develop. 2010; 5: 14 .

99. Lai CSL, Gerrelli D, Monaco AP, Fisher SE, Copp AJ. FOXP2 expression during brain development coincides with adult sites of pathology in a severe speech and language disorder. Brain J Neurol. 2003; 126: 2455-62.

100. Belle M, Godefroy D, Couly G, Malone SA, Collier F, Giacobini P, Chédotal A. Tridimensional Visualization and Analysis of Early Human Development. Cell. 2017; 169: 161-173.e12.

101. Bowers JM, Konopka G. The role of the FOXP family of transcription factors in ASD. Dis Markers. 2012; 33: 25160 .

102. Bowers JM, Perez-Pouchoulen M, Edwards NS, McCarthy MM. Foxp2 mediates sex differences in ultrasonic vocalization by rat pups and directs order of maternal retrieval. J Neurosci Off J Soc Neurosci. 2013; 33: 3276-83.

103. Fujita E, Tanabe Y, Shiota A, Ueda M, Suwa K, Momoi MY, Momoi T. Ultrasonic vocalization impairment of Foxp2 $(\mathrm{R} 552 \mathrm{H})$ knockin mice related to speech-language disorder and abnormality of Purkinje cells. Proc Natl Acad Sci U S A. 2008; 105: 3117-22.

104. Lischinsky JE, Sokolowski K, Li P, Esumi S, Kamal Y, Goodrich M, Oboti L, Hammond TR, Krishnamoorthy M, Feldman D, Huntsman M, Liu J, Corbin JG. Embryonic transcription factor expression in mice predicts medial amygdala neuronal identity and sex-specific responses to innate behavioral cues. eLife. 2017; 6 .

105. Bowers JM, Perez-Pouchoulen M, Roby CR, Ryan TE, McCarthy MM. Androgen Modulation of Foxp1 and Foxp2 in the Developing Rat Brain: Impact on Sex Specific Vocalization. Endocrinology. 2014; 155: 4881-94.

106. Walker RM, Hill AE, Newman AC, Hamilton G, Torrance HS, Anderson SM, Ogawa F, Derizioti P, Nicod J, Vernes SC, Fisher SE, Thomson PA, Porteous DJ, et al. The DISC1 promoter: characterization and regulation by FOXP2. Hum Mol Genet. 2012; 21: 2862-72.

107. Konopka G, Friedrich T, Davis-Turak J, Winden K, Oldham MC, Gao F, Chen L, Wang G-Z, Luo R, Preuss TM, Geschwind DH. Human-specific transcriptional networks in the brain. Neuron. 2012; 75: 601-17.

108. Chiu Y-C, Li M-Y, Liu Y-H, Ding J-Y, Yu J-Y, Wang T-W.
Foxp2 regulates neuronal differentiation and neuronal subtype specification. Dev Neurobiol. 2014; 74: 723-38.

109. Devanna P, Middelbeek J, Vernes SC. FOXP2 drives neuronal differentiation by interacting with retinoic acid signaling pathways. Front Cell Neurosci. 2014; 8: 305.

110. Mukamel Z, Konopka G, Wexler E, Osborn GE, Dong H, Bergman MY, Levitt P, Geschwind DH. Regulation of MET by FOXP2, genes implicated in higher cognitive dysfunction and autism risk. J Neurosci Off J Soc Neurosci. 2011; 31: 11437-42.

111. Sia GM, Clem RL, Huganir RL. The human languageassociated gene SRPX2 regulates synapse formation and vocalization in mice. Science. 2013; 342: 987-91.

112. Zhan T, Rindtorff N, Boutros M. Wnt signaling in cancer. Oncogene. 2017; 36: 1461-73.

113. Shu W, Yang H, Zhang L, Lu MM, Morrisey EE. Characterization of a new subfamily of winged-helix/ forkhead (Fox) genes that are expressed in the lung and act as transcriptional repressors. J Biol Chem. 2001; 276: 27488-97.

114. Burraco AB. Genes y lenguaje: aspectos ontogenéticos, filogenéticos y cognitivos. Reverte; 2009. 498 p.

115. Daudigeos-Dubus E, Le Dret L, Bawa O, Opolon P, Vievard A, Villa I, Bosq J, Vassal G, Geoerger B. Dual inhibition using cabozantinib overcomes HGF/MET signaling mediated resistance to pan-VEGFR inhibition in orthotopic and metastatic neuroblastoma tumors. Int J Oncol. 2017; 50: 203-11.

116. Hung YP, Lee JP, Bellizzi AM, Hornick JL. PHOX2B reliably distinguishes neuroblastoma among small round blue cell tumours. Histopathology. 2017.

117. Kasim M, Heß V, Scholz H, Persson PB, Fähling M. Achaete-Scute Homolog 1 Expression Controls Cellular Differentiation of Neuroblastoma. Front Mol Neurosci. 2016; 9: 156.

118. Briggs KJ, Corcoran-Schwartz IM, Zhang W, Harcke T, Devereux WL, Baylin SB, Eberhart CG, Watkins DN. Cooperation between the Hic1 and Ptch1 tumor suppressors in medulloblastoma. Genes Dev. 2008; 22: 770-85.

119. Tu M, Cai L, Zheng W, Su Z, Chen Y, Qi S. CD164 regulates proliferation and apoptosis by targeting PTEN in human glioma. Mol Med Rep. 2017; 15: 1713-21.

120. Muley PD, McNeill EM, Marzinke MA, Knobel KM, Barr MM, Clagett-Dame M. The atRA-responsive gene neuron navigator 2 functions in neurite outgrowth and axonal elongation. Dev Neurobiol. 2008; 68: 1441-53.

121. Simpkins AN, Elmarakby AA, Quigley JE, Schreihofer DA, Hammock BD, Imig JD. Soluble Epoxide Hydrolase Inhibition Modulates Gene Expression of Anti-Apoptotic and Apoptotic Factors. FASEB JournalI. 2008; 22: 733.11.

122. Dmitrenko VV, Bojko OI, Shostak KO, Vitak NY, Bukreeva TV, Rozumenko VD, Malysheva TA, Shamayev MI, Zozulya YP, Kavsan VM. Characterization of genes, downregulated in human glioma, potential tumor suppressor 
genes. Biopolym Cell. 2007; 23: 347-62.

123. Kumar S. The Role of Tumour Suppressor Tyrosine Kinase SYK in Glioblastoma and Breast Cancer. Universitätsbibliothek der TU München; 2008. book p.

124. Gorbunova V, Seluanov A, Zhang Z, Gladyshev VN, Vijg J. Comparative genetics of longevity and cancer: insights from long-lived rodents. Nat Rev Genet. 2014; 15: 531-40.

125. Weaver I, Sakurai T, Buxbaum, JD, Rymar, V, Sadikot, A, Kaplan, DR, Miller, FD. The speech vocalization gene, foxp2, regulates interneuron fate decisions. Front Neurosci Conf Abstr BRAIN Platf Physiol Poster Day. 2009.

126. Clovis YM, Enard W, Marinaro F, Huttner WB, De Pietri Tonelli D. Convergent repression of Foxp2 3'UTR by miR9 and miR-132 in embryonic mouse neocortex: implications for radial migration of neurons. Dev Camb Engl. 2012; 139: 3332-42.

127. Reimers-Kipping S, Hevers W, Pääbo S, Enard W. Humanized Foxp2 specifically affects cortico-basal ganglia circuits. Neuroscience. 2011; 175: 75-84.

128. Schulz SB, Haesler S, Scharff C, Rochefort C. Knockdown of FoxP2 alters spine density in Area X of the zebra finch. Genes Brain Behav. 2010; 9: 732-40.

129. Nudel R, Newbury DF. FOXP2. Wiley Interdiscip Rev Cogn Sci. 2013; 4: 547-60.

130. Lennon PA, Cooper ML, Peiffer DA, Gunderson KL, Patel A, Peters S, Cheung SW, Bacino CA. Deletion of 7q31.1 supports involvement of FOXP2 in language impairment: clinical report and review. Am J Med Genet A. 2007; 143A: 791-8.

131. MacDermot KD, Bonora E, Sykes N, Coupe A-M, Lai CSL, Vernes SC, Vargha-Khadem F, McKenzie F, Smith RL, Monaco AP, Fisher SE. Identification of FOXP2 truncation as a novel cause of developmental speech and language deficits. Am J Hum Genet. 2005; 76: 1074-80.

132. Shriberg LD, Ballard KJ, Tomblin JB, Duffy JR, Odell KH, Williams CA. Speech, prosody, and voice characteristics of a mother and daughter with a 7;13 translocation affecting FOXP2. J Speech Lang Hear Res JSLHR. 2006; 49: 500-25.

133. Zeesman S, Nowaczyk MJM, Teshima I, Roberts W, Cardy JO, Brian J, Senman L, Feuk L, Osborne LR, Scherer SW. Speech and language impairment and oromotor dyspraxia due to deletion of $7 \mathrm{q} 31$ that involves FOXP2. Am J Med Genet A. 2006; 140: 509-14.

134. Graham SA, Fisher SE. Decoding the genetics of speech and language. Curr Opin Neurobiol. 2013; 23: 43-51.

135. Deriziotis P, O'Roak BJ, Graham SA, Estruch SB, Dimitropoulou D, Bernier RA, Gerdts J, Shendure J, Eichler EE, Fisher SE. De novo TBR1 mutations in sporadic autism disrupt protein functions. Nat Commun. 2014; 5: ncomms5954.

136. Peñagarikano O, Geschwind DH. What does CNTNAP2 reveal about autism spectrum disorder? Trends Mol Med. 2012; 18: 156-63.

137. Khanzada NS, Butler MG, Manzardo AM. GeneAnalytics
Pathway Analysis and Genetic Overlap among Autism Spectrum Disorder, Bipolar Disorder and Schizophrenia. Int J Mol Sci. 2017; 18: 527.

138. Španiel F, Horáček J, Tintěra J, Ibrahim I, Novák T, Čermák J, Klírová M, Höschl C. Genetic variation in FOXP2 alters grey matter concentrations in schizophrenia patients. Neurosci Lett. 2011; 493: 131-5.

139. Groszer M, Keays DA, Deacon RMJ, de Bono JP, PrasadMulcare S, Gaub S, Baum MG, French CA, Nicod J, Coventry JA, Enard W, Fray M, Brown SDM, et al. Impaired synaptic plasticity and motor learning in mice with a point mutation implicated in human speech deficits. Curr Biol CB. 2008; 18: 354-62.

140. Padovani A, Cosseddu M, Premi E, Archetti S, Papetti A, Agosti C, Bigni B, Cerini C, Paghera B, Bellelli G, Borroni $\mathrm{B}$. The speech and language FOXP2 gene modulates the phenotype of frontotemporal lobar degeneration. $\mathrm{J}$ Alzheimers Dis JAD. 2010; 22: 923-31.

141. Tsui D, Vessey JP, Tomita H, Kaplan DR, Miller FD. FoxP2 regulates neurogenesis during embryonic cortical development. J Neurosci Off J Soc Neurosci. 2013; 33: 244 58.

142. Cuiffo BG, Karnoub AE. Silencing FOXP2 in breast cancer cells promotes cancer stem cell traits and metastasis. Mol Cell Oncol [Internet]. 2015 [cited 2017 Jun 17]; 3.

143. Xu S, Wang T, Song W, Jiang T, Zhang F, Yin Y, Jiang S-W, Wu K, Yu Z, Wang C, Chen K. The inhibitory effects of AR/ miR-190a/YB-1 negative feedback loop on prostate cancer and underlying mechanism. Sci Rep. 2015; 5: 13528.

144. Formosa A, Lena AM, Markert EK, Cortelli S, Miano R, Mauriello A, Croce N, Vandesompele J, Mestdagh P, Finazzi-Agrò E, Levine AJ, Melino G, Bernardini S, et al. DNA methylation silences miR-132 in prostate cancer. Oncogene. 2013; 32: 127-34.

145. Song $\mathrm{X}$, Tang, Y, Lei, XH, Zhao, SC, Wu, ZQ. miR618 Inhibits Prostate Cancer Migration and Invasion by Targeting FOXP2. J Cancer. 2017; 8: 2501-10.

146. Seznec J, Weit S, Naumann U. Gene expression profile in a glioma cell line resistant to cell death induced by the chimeric tumor suppressor-1 (CTS-1), a dominant-positive variant of p53--the role of NFkappaB. Carcinogenesis. 2010; 31: 411-8.

147. Seznec J, Naumann U. Microarray analysis in a cell death resistant glioma cell line to identify signaling pathways and novel genes controlling resistance and malignancy. Cancers. $2011 ; 3: 2827-43$.

148. Tang H, Guo Q, Zhang C, Zhu J, Yang H, Zou Y-L, Yan Y, Hong D, Sou T, Yan X-M. Identification of an intermediate signature that marks the initial phases of the colorectal adenoma-carcinoma transition. Int J Mol Med. 2010; 26: 631.

149. Gascoyne DM, Spearman H, Lyne L, Puliyadi R, PerezAlcantara M, Coulton L, Fisher SE, Croucher PI, Banham AH. The Forkhead Transcription Factor FOXP2 Is Required 
for Regulation of $\mathrm{p} 21 \mathrm{WAF} 1 / \mathrm{CIP} 1$ in 143B Osteosarcoma Cell Growth Arrest. PloS One. 2015; 10: e0128513.

150. Zhang W, Duan N, Song T, Li Z, Zhang C, Chen X. The Emerging Roles of Forkhead Box (FOX) Proteins in Osteosarcoma. J Cancer. 2017; 8: 1619-28.

151. Cai C, Qin X, Wu Z, Shen Q, Yang W, Zhang S, Duan J, Liang F, Liu C. Inhibitory effect of MyoD on the proliferation of breast cancer cells. Oncol Lett. 2016; 11: 3589-96.

152. Dey J, Dubuc AM, Pedro KD, Thirstrup D, Mecham B, Northcott PA, Wu X, Shih D, Tapscott SJ, LeBlanc M, Taylor MD, Olson JM. MyoD is a tumor suppressor gene in medulloblastoma. Cancer Res. 2013; 73: 6828-37.

153. Shern JF, Chen L, Chmielecki J, Wei JS, Patidar R, Rosenberg M, Ambrogio L, Auclair D, Wang J, Song YK, Tolman C, Hurd L, Liao H, et al. Comprehensive genomic analysis of rhabdomyosarcoma reveals a landscape of alterations affecting a common genetic axis in fusionpositive and fusion-negative tumors. Cancer Discov. 2014; 4: 216-31.

154. Pei X-H, Lv X-Q, Li H-X. Sox5 induces epithelial to mesenchymal transition by transactivation of Twist1. Biochem Biophys Res Commun. 2014; 446: 322-7.

155. David-Cordonnier M-H, Hamdane M, Bailly C, D’Halluin J-C. Determination of the human c-Abl consensus DNA binding site. FEBS Lett. 1998; 424: 177-82.

156. Mertin S, McDowall SG, Harley VR. The DNA-binding specificity of SOX9 and other SOX proteins. Nucleic Acids Res. 1999; 27: 1359-64.

157. Gupta R, Toufaily C, Annabi B. Caveolin and cavin family members: dual roles in cancer. Biochimie. 2014; 107 Pt B: 188-202.

158. Suzuki N, Yoshioka N, Uekawa A, Matsumura N, Tozawa A, Koike J, Konishi I, Kiguchi K, Ishizuka B. Transcription factor POU6F1 is important for proliferation of clear cell adenocarcinoma of the ovary and is a potential new molecular target. Int J Gynecol Cancer Off J Int Gynecol Cancer Soc. 2010; 20: 212-9.

159. Ayub Q, Yngvadottir B, Chen Y, Xue Y, Hu M, Vernes SC, Fisher SE, Tyler-Smith C. FOXP2 targets show evidence of positive selection in European populations. Am J Hum Genet. 2013; 92: 696-706.

160. Scherer SW, Cheung J, MacDonald JR, Osborne LR, Nakabayashi K, Herbrick J-A, Carson AR, ParkerKatiraee L, Skaug J, Khaja R, Zhang J, Hudek AK, Li M, et al. Human chromosome 7: DNA sequence and biology. Science. 2003; 300: 767-72.

161. Kalkman HO. A review of the evidence for the canonical Wnt pathway in autism spectrum disorders. Mol Autism. 2012; 3: 10

162. Shigekawa T, Ijichi N, Ikeda K, Horie-Inoue K, Shimizu C, Saji S, Aogi K, Tsuda H, Osaki A, Saeki T, Inoue S. FOXP1, an Estrogen-Inducible Transcription Factor, Modulates Cell Proliferation in Breast Cancer Cells and 5-Year Recurrence-
Free Survival of Patients with Tamoxifen-Treated Breast Cancer. Horm Cancer. 2011; 2: 286-97.

163. Organ SL, Tsao M-S. An overview of the c-MET signaling pathway. Ther Adv Med Oncol. 2011; 3: S7-19.

164. Langford PR, Keyes L, Hansen MDH. Plasma membrane ion fluxes and NFAT-dependent gene transcription contribute to c-met-induced epithelial scattering. J Cell Sci. 2012; 125: 4001-13.

165. Ho L, Crabtree G. A Foxy tango with NFAT. Nat Immunol. 2006; 7: 906-8.

166. Wu Y, Borde M, Heissmeyer V, Feuerer M, Lapan AD, Stroud JC, Bates DL, Guo L, Han A, Ziegler SF, Mathis D, Benoist $\mathrm{C}$, Chen $\mathrm{L}$, et al. FOXP3 controls regulatory $\mathrm{T}$ cell function through cooperation with NFAT. Cell. 2006; 126: 375-87.

167. Zhou Z, Song X, Li B, Greene MI. FOXP3 and its partners: structural and biochemical insights into the regulation of FOXP3 activity. Immunol Res. 2008; 42: 19-28.

168. Nastase A, Pâslaru L, Niculescu AM, Ionescu M, Dumitraşcu T, Herlea V, Dima S, Gheorghe C, Lazar V, Popescu I. Prognostic and predictive potential molecular biomarkers in colon cancer. Chir Buchar Rom 1990. 2011; 106: 177-85.

169. Lee J-Y, Hur H, Yun HJ, Kim Y, Yang S, Kim SI, Kim MH. HOXB5 Promotes the Proliferation and Invasion of Breast Cancer Cells. Int J Biol Sci. 2015; 11: 701-11.

170. Zhang B, Li N, Zhang H. Knockdown of Homeobox B5 (HOXB5) Inhibits Cell Proliferation, Migration, and Invasion in Non-Small Cell Lung Cancer Cells Through Inactivation of the Wnt/ $\beta$-Catenin Pathway. Oncol Res. 2017.

171. Bernard D, Vindrieux D. PLA2R1: expression and function in cancer. Biochim Biophys Acta. 2014; 1846: 40-4.

172. Dlugosz A, Janecka A. ABC Transporters in the Development of Multidrug Resistance in Cancer Therapy. Curr Pharm Des. 2016; 22: 4705-16.

173. Hedditch EL, Gao B, Russell AJ, Lu Y, Emmanuel C, Beesley J, Johnatty SE, Chen X, Harnett P, George J, Australian Ovarian Cancer Study Group, Williams RT, Flemming $\mathrm{C}$, et al. ABCA transporter gene expression and poor outcome in epithelial ovarian cancer. J Natl Cancer Inst. 2014; 106.

174. Mao Q, Unadkat JD. Role of the breast cancer resistance protein (BCRP/ABCG2) in drug transport--an update. AAPS J. 2015; 17: 65-82.

175. Enard W, Przeworski M, Fisher SE, Lai CSL, Wiebe V, Kitano T, Monaco AP, Pääbo S. Molecular evolution of FOXP2, a gene involved in speech and language. Nature. 2002; 418: 869-72.

176. Newbury DF, Bonora E, Lamb JA, Fisher SE, Lai CSL, Baird G, Jannoun L, Slonims V, Stott CM, Merricks MJ, Bolton PF, Bailey AJ, Monaco AP. FOXP2 Is Not a Major Susceptibility Gene for Autism or Specific Language Impairment. Am J Hum Genet. 2002; 70: 1318-27. 
177. Bruce HA, Margolis RL. FOXP2: novel exons, splice variants, and CAG repeat length stability. Hum Genet. 2002; 111: 136-44.

178. Chen D-T, Nasir A, Culhane A, Venkataramu C, Fulp W, Rubio R, Wang T, Agrawal D, McCarthy SM, Gruidl M, Bloom G, Anderson T, White J, et al. Proliferative genes dominate malignancy-risk gene signature in histologicallynormal breast tissue. Breast Cancer Res Treat. 2010; 119: 335-46. 